Pacific

Journal of

Mathematics

FORMAL DEFORMATIONS OF POISSON STRUCTURES IN LOW DIMENSIONS

AnNe Pichereau

Volume 239 No. 1

January 2009 


\title{
FORMAL DEFORMATIONS OF POISSON STRUCTURES IN LOW DIMENSIONS
}

\author{
ANNE PICHEREAU
}

\begin{abstract}
In this paper, we study formal deformations of Poisson structures, especially for two families of Poisson varieties in dimensions two and three. For these families of Poisson structures, using an explicit basis of the second Poisson cohomology space, we solve the deformation equations at each step and obtain a large family of formal deformations for each Poisson structure that we consider. With the help of an explicit formula, we show that this family contains, modulo equivalence, all possible formal deformations. We show moreover that, when the Poisson structure is generic, all members of the family are nonequivalent.
\end{abstract}

1. Introduction

105

2. Conditions for a system of representatives for all formal deformations

3. Formal deformations of Poisson structures in dimension two and three

4. Final remarks

Acknowledgments

References

\section{Introduction}

D. Poisson introduced his eponymous structure into classical mechanics when, in 1809 , he discovered the natural symplectic structure on $\mathbb{R}^{2 r}$. This structure permits one to write Hamilton's equations in a more natural way, with positions

MSC2000: 17B63, 58H15, 17B55.

Keywords: Deformations, Poisson structures, Poisson cohomology.

The author was supported by a grant of the research network LIEGRITS, in the Marie Curie Research Training Network MRTN-CT 2003-505078, and a grant of the EPDI (European Post-Doctoral Institute). 
and momenta playing symmetric roles. This symplectic structure is, in a sense, the most simple example of a Poisson structure, and it takes the form

$$
\{F, G\}=\sum_{i=1}^{r}\left(\frac{\partial F}{\partial q_{i}} \frac{\partial G}{\partial p_{i}}-\frac{\partial F}{\partial p_{i}} \frac{\partial G}{\partial q_{i}}\right),
$$

for smooth functions $F$ and $G$ on $\mathbb{R}^{2 r}$. In 1839, C. Jacobi showed that this bracket satisfies the (Jacobi) identity

$$
\{\{F, G\}, H\}+\{\{G, H\}, F\}+\{\{H, F\}, G\}=0,
$$

thereby explaining Poisson's theorem: The bracket of two constants of motion is a constant of motion. In general, one defines a Poisson structure on an associative commutative algebra $(\mathscr{A}, \cdot)$ over a field $\mathbb{F}$ as being a Lie algebra structure $\{\cdot, \cdot\}: \mathscr{A} \times \mathscr{A} \rightarrow \mathscr{A}$ that is a biderivation of $\mathscr{A}$, that is, it satisfies the derivation property in each of its arguments:

$$
\{F \cdot G, H\}=F \cdot\{G, H\}+G \cdot\{F, H\} \quad \text { for all } F, G, H \in A \text {. }
$$

A smooth manifold $M$ is said to be a Poisson manifold if its algebra of smooth functions $C^{\infty}(M)$ is equipped with a Poisson structure.

Poisson structures are also inherent in quantum mechanics, since it was observed by P. Dirac that, up to a factor $2 i \pi / h$, the commutator of observables, appearing in the work of W. Heisenberg, is the analogue of the Poisson bracket (1) of classical mechanics. They also play an important role in the theory of deformation quantization, which is linked to quantum mechanics, as shown in [Bayen et al. 1978a; 1978b]. Translated in a mathematical language, this theory is the study of deformations of associative, commutative algebras. In 1997, M. Kontsevich proved that, given a Poisson manifold $(M,\{\cdot, \cdot\})$, the equivalence classes of formal deformations of the Poisson structure $\{\cdot, \cdot\}$ correspond to the equivalence classes of those formal deformations of the associative product of $C^{\infty}(M)$ that have as first order term the bracket $\{\cdot, \cdot\}$ This result underlies the importance of formal deformations of Poisson structures, which is the subject of this paper.

Let $(\mathscr{A},\{\cdot, \cdot\})$ be a Poisson algebra over $\mathbb{F}$. A formal deformation of $\{\cdot, \cdot\}$ (see [Sternheimer 1998] and [Gutt 2000]) is a map $\pi_{*}: \mathscr{A}[[v]] \times \mathscr{A}[[v]] \rightarrow \mathscr{A}[[v]]$ that extends $\{\cdot, \cdot\}$ as

$$
\pi_{*}=\{\cdot, \cdot\}+\pi_{1} v+\pi_{2} v^{2}+\cdots+\pi_{n} v^{n}+\cdots,
$$

where each map $\pi_{i}: \mathscr{A} \times \mathscr{A} \rightarrow \mathscr{A}$ is a skew-symmetric biderivation of $\mathscr{A}$, and makes $\left(\mathscr{A}[[v]], \pi_{*}\right)$ into a Poisson algebra over the ring $\mathbb{E}[[v]]$, where the associative product on $\mathscr{A}[[v]]$ is the one inherited from the initial one on $\mathscr{A}$. To simplify the notation and to emphasize the fact that the Poisson structure $\{\cdot, \cdot\}$ is the first 
term of $\pi_{*}$, we also denote it by $\pi_{0}$. Notice that, in similarity to the associative product, each skew-symmetric biderivation of $\mathscr{A}$ (like the $\pi_{i}$ ) can be seen as a map $\mathscr{A}[[v]] \times \mathscr{A}[[v]] \rightarrow \mathscr{A}[[v]]$ by considering its extension by $\mathbb{E}[[v]]$-bilinearity. In particular, such an extension of $\pi_{0}$ is a formal deformation of $\pi_{0}=\{\cdot, \cdot\}$, but we stress that our goal is to consider all formal deformations of $\pi_{0}$ and not only the one obtained in this way. If one works modulo $v^{n+1}$, then one speaks of an $n$-th order deformation. Deformations are always studied up to equivalence, two formal deformations $\pi_{*}$ and $\pi_{*}^{\prime}$ being equivalent if there exists a morphism $\Phi:\left(\mathscr{A}[[v]], \pi_{*}\right) \rightarrow\left(\mathscr{A}[[v]], \pi_{*}^{\prime}\right)$ of Poisson algebras over $\mathbb{F}[[v]]$ that is the identity modulo $v$.

Studying deformations of a Poisson structure $\{\cdot, \cdot\}$ means studying the following questions:

Question 1 (rigidity). Do there exist nontrivial formal deformations of $\{\cdot, \cdot\}$ ?

Question 2 (extendibility). Given an $n$-th order deformation of $\{\cdot, \cdot\}$, does it extend to an $(n+1)$-st order deformation?

Question 3 (formula). Is it possible to obtain an explicit formula for all formal $n$-th order deformations of $\{\cdot, \cdot\}$ (up to equivalence)?

Question 4 (properties). Which properties of the Poisson bracket $\{\cdot, \cdot\}$ are stable under deformation?

In general, the deformation theory of a structure (an associative or a Lie product, for example) is governed by an associated cohomology, which provides some tools for giving an answer to Questions 1-4. In the particular Poisson case, the cohomology that plays this role is Poisson cohomology (introduced in [Lichnerowicz 1977], but see also [Huebschmann 1990] for an algebraic approach). For a Poisson algebra $\left(\mathscr{A}, \pi_{0}=\{\cdot, \cdot\}\right)$, the Poisson complex (which will be explained in Section 2.1) is defined on the space $\mathfrak{X}^{\bullet}(\mathscr{A})$ of all skew-symmetric multiderivations of $\mathscr{A}$ (in particular, $\pi_{0} \in \mathfrak{X}^{2}(\mathscr{A})$ ). For $k \in \mathbb{N}$, the $k$-th Poisson cohomology space is then denoted by $H^{k}\left(\mathscr{A}, \pi_{0}\right)$.

As we will see in Section 2.1, the third Poisson cohomology space $H^{3}\left(\mathscr{A}, \pi_{0}\right)$ appears naturally in the construction of formal deformations of $\pi_{0}$ : a map of the form $\pi_{*}=\sum_{n \in \mathbb{N}} \pi_{n} \nu^{n}: \mathscr{A}[[v]] \times \mathscr{A}[[v]] \rightarrow \mathscr{A}[[v]]$ is a formal deformation of $\pi_{0}$ if and only if each $\pi_{n}$ for $n \in \mathbb{N}^{*}$ is a skew-symmetric biderivation of $\mathscr{A}$ that satisfies a certain cohomological equation in $H^{3}\left(\mathscr{A}, \pi_{0}\right)$. That is why one refers to $H^{3}\left(\mathscr{A}, \pi_{0}\right)$ as being the set of obstructions to deformations of $\pi_{0}$. The second Poisson cohomology space $H^{2}\left(\mathscr{A}, \pi_{0}\right)$ plays also a fundamental role in this study. Indeed, if $\pi_{n} \in \mathfrak{X}^{2}(\mathscr{A})$ is a solution of the equation mentioned above, then $\pi_{n}^{\prime}=\pi_{n}+P$, where $P$ is any 2-cocycle, is also a solution, but if in particular $P$ is a 2-coboundary, then the corresponding $\pi_{n}^{\prime}$ gives rise to an ( $n$-th order) deformation, equivalent to the 
one obtained with $\pi_{n}$. Hence, the choice at each step of the construction of $\pi_{*}$ is a choice in $H^{2}\left(\mathscr{A}, \pi_{0}\right)$. The difficulty for constructing a formal deformation of $\pi_{0}$ can now be explained as follows: Even if, at one step, one finds a solution to the cohomological equation mentioned above, the choice (in $H^{2}\left(\mathscr{A}, \pi_{0}\right)$ ) that one has to make at this step changes the cohomological equations (in $H^{3}\left(\mathscr{A}, \pi_{0}\right)$ ) that one will have to solve at each of the following steps. Now, depending on the choices that have been done previously, the cohomological equation at one step can even be solvable or not! This explains why, in general, it is difficult, even with a precise knowledge of the corresponding cohomology, to answer Questions 1-4.

In Section 2, we prove a proposition that gives, for a class of Poisson structures, a system of representatives for all formal deformations, modulo equivalence. We formulate it here for the case of formal deformations, even if it is equally valid for the case of $n$-th order deformations.

Proposition 1.1. Let $\left(\mathscr{A}, \pi_{0}\right)$ be a Poisson algebra. Denote by $\left(\vartheta_{k} \in \mathfrak{X}^{2}(\mathscr{A})\right)_{k \in \mathscr{K}} a$ set of 2-cocycles whose cohomology classes form a basis of $H^{2}\left(\mathscr{A}, \pi_{0}\right)$. Define $\mathscr{Y}$ to be the set of all $\boldsymbol{a}=\left(a_{n}^{k} \in \mathbb{F}\right)_{k \in \mathscr{K}, n \in \mathbb{N}^{*}}$ such that, for every $n_{0} \in \mathbb{N}^{*}$, the sequence $\left(a_{n_{0}}^{k}\right)_{k \in \mathscr{K}}$ has a finite support.

Suppose that to each element a of $\mathscr{S}$ is associated a sequence $\left(\Psi_{n}^{a} \in \mathfrak{X}^{2}(\mathscr{A})\right)_{n \in \mathbb{N}^{*}}$ of skew-symmetric biderivations of $A$ with these properties:

- The skew-symmetric biderivation $\Psi_{1}^{a}$ of $\mathscr{A}$ is zero, that is, $\Psi_{1}^{a}=0$.

- For all $n \in \mathbb{N}^{*}, \Psi_{n}^{a}$ only depends on the $a_{m}^{k}$ with $k \in \mathscr{K}$ and $1 \leq m \leq n-1$.

- The skew-symmetric biderivation of $A[[v]]$ defined by

$$
\pi_{*}^{a}:=\pi_{0}+\sum_{n \in \mathbb{N}^{*}}\left(\Psi_{n}^{a}+\sum_{k \in \mathscr{K}} a_{n}^{k} \vartheta_{k}\right) v^{n}
$$

is a formal deformation of $\pi_{0}$.

Then

(a) for every formal deformation $\pi_{*}$ of $\pi_{0}$, there exists an element a of 9 such that $\pi_{*}$ is equivalent to $\pi_{*}^{a}$;

(b) if, in addition, the first Poisson cohomology space $H^{1}\left(\mathcal{A}, \pi_{0}\right)$ is zero, then the element a from (a) is unique.

We stress that this proposition involves not only the space $H^{3}\left(\mathscr{A}, \pi_{0}\right)$ (implicitly in the existence of the family $\left.\left(\pi_{*}^{a}\right)_{a \in \mathscr{S}}\right)$ and the space $H^{2}\left(\mathscr{A}, \pi_{0}\right)$ (explicitly in the writing of the family $\left.\left(\pi_{*}^{a}\right)_{\boldsymbol{a} \in \mathscr{S}}\right)$, but also $H^{1}\left(\mathscr{A}, \pi_{0}\right)$.

The hypotheses in Proposition 1.1 are strong, but in Section 3, we will show that they are satisfied for several large families of Poisson structures in low dimensions. We will do that, for each family, by using an explicit basis of $H^{2}\left(\mathscr{A}, \pi_{0}\right)$ and by 
constructing an explicit formula for suitable $\Psi_{n}^{a}$, which means solving the cohomological equations in $H^{3}\left(\mathscr{A}, \pi_{0}\right)$ that govern the extendibility of deformations.

We first consider a big family of Poisson structures that equip $\mathscr{A}:=\mathbb{F}[x, y, z]$, the algebra of regular (polynomial) functions on the three-dimensional affine space $\mathbb{F}^{3}$. Indeed, to each polynomial $\varphi \in \mathscr{A}$, one can associate a Poisson structure $\{\cdot, \cdot\}_{\varphi}$ on $\mathscr{A}$, defined by the brackets

$$
\{x, y\}_{\varphi}=\frac{\partial \varphi}{\partial z}, \quad\{y, z\}_{\varphi}=\frac{\partial \varphi}{\partial x}, \quad\{z, x\}_{\varphi}=\frac{\partial \varphi}{\partial y} .
$$

Note that this Poisson structure appears for example as the Poisson structure transverse to a subregular nilpotent orbit of a Lie algebra; see [Damianou et al. 2007].

In [Pichereau 2006], we have already obtained explicit bases for the spaces $H^{1}\left(\mathscr{A},\{\cdot, \cdot\}_{\varphi}\right)$ and $H^{2}\left(\mathscr{A},\{\cdot, \cdot\}_{\varphi}\right)$, in case the polynomial $\varphi$ is (weight) homogeneous with an isolated singularity, that is, when the surface $\mathscr{F}_{\varphi}:\{\varphi=0\}$ (the singular locus of $\varphi$ ) is given by a (weight) homogeneous equation and admits an isolated singularity (at the origin). In Section 3, we will use these results to show that, after a change of basis of $H^{2}\left(\mathscr{A},\{\cdot, \cdot\}_{\varphi}\right)$, we are able to exhibit a family of skew-symmetric biderivations $\Psi_{n}^{a}$ of $\mathscr{A}$ that satisfy the conditions of Proposition 1.1. Since we obtain in fact an explicit formula for every $\Psi_{n}^{a}$, this proposition permits us to write an explicit formula for all formal deformations of $\{\cdot, \cdot\}_{\varphi}$, up to equivalence. More precisely, we have the following proposition (see Proposition 3.3), given here in a formal context although it is also valid for $n$-th order deformations.

Proposition 1.2. Let $\varphi \in \mathscr{A}=\mathbb{F}[x, y, z]$ be a weight-homogeneous polynomial with an isolated singularity. Consider the Poisson algebra $\left(\mathscr{A},\{\cdot, \cdot\}_{\varphi}\right)$, where $\{\cdot, \cdot\}_{\varphi}$ is the Poisson bracket given by (4).

(a) There exist skew-symmetric biderivations $\Psi_{n}^{a}$ of $\mathscr{A}$ (for which we have explicit formulas) satisfying the hypotheses of Proposition 1.1 for $\left(\mathscr{A},\{\cdot, \cdot\}_{\varphi}\right)$.

(b) The Poisson algebra $\left(\mathscr{A},\{\cdot, \cdot\}_{\varphi}\right)$ satisfies the conditions of Proposition 1.1(b) unless the (weighted) degree of $\varphi$ equals the sum of the weights of the variables $x, y$ and $z$.

At that point, we have obtained a clear answer to Questions 1 and 3 above (those of rigidity and formula). Because Proposition 1.1 is also true for $n$-th order deformations, we also have an answer to Question 2 (extendibility): Every n-th order deformation of $\{\cdot, \cdot\}_{\varphi}$ extends to an $(n+1)$-st order deformation (Corollary 3.5$)$.

Finally, using the explicit formula mentioned above (for all formal deformations of the bracket $\{\cdot, \cdot\}_{\varphi}$ ), we will also give a partial answer to Question 4: The formal deformations of $\{\cdot, \cdot\}_{\varphi}$ all admit formal Casimirs (Corollary 3.8). 
The polynomial $\varphi \in \mathbb{E}[x, y, z]$ is a Casimir for the Poisson structure $\{\cdot, \cdot\}_{\varphi}$, so this Poisson structure restricts to a Poisson structure $\{\cdot, \cdot\}_{\mathscr{A}_{\varphi}}$ on the quotient algebra $\mathscr{A}_{\varphi}:=\mathbb{F}[x, y, z] /\langle\varphi\rangle$, which is the algebra of regular functions on the surface $\mathscr{F}_{\varphi}:\{\varphi=0\} \subset \mathbb{F}^{3}$. The deformations of the Poisson structure $\{\cdot, \cdot\}_{\mathscr{A}_{\varphi}}$ are studied in Section 3.5. In fact, under the previous hypotheses on $\varphi$, the cohomological equations mentioned above are in this case trivial and this fact, together with an explicit basis of the second Poisson cohomology space (obtained in [Pichereau 2006]), permit us to give an explicit formula for all formal deformations of $\{\cdot, \cdot\}_{\mathscr{A}_{\varphi}}$, up to equivalence (see Proposition 3.9).

\section{Conditions for a system of representatives for all formal deformations}

In this part, we want to prove Proposition 1.1. To do that, we will need several intermediate results. These will be proved in an elementary way, in the sense that our proofs will only need the properties of the Schouten bracket and the definition of the Poisson cohomology, as recalled in Section 2.1.

2.1. Preliminaries. In this paper, $\mathbb{F}$ is an arbitrary field of characteristic zero. We recall that a Poisson structure $\{\cdot, \cdot\}$ (which is also denoted by $\pi_{0}$ ) on an associative commutative algebra $(\mathscr{A}, \cdot)$ is a skew-symmetric biderivation of $\mathscr{A}$, that is, a map $\{\cdot, \cdot\}: \bigwedge^{2} \mathscr{A} \rightarrow \mathscr{A}$ that satisfies the derivation property

$$
\{F G, H\}=F\{G, H\}+G\{F, H\} \quad \text { for all } F, G, H \in \mathscr{A}
$$

(where $F G$ stands for $F \cdot G$ ), and that is also a Lie structure on $\mathscr{A}$, that is, the map also satisfies the Jacobi identity.

We denote by $\mathbb{F}^{v}$ the ring of all formal power series in an indeterminate $v$ and with coefficients in $\mathbb{F}$, that is, $\mathbb{F}^{v}:=\mathbb{F}[[v]]$. We will also consider the $\mathbb{F}^{v}$-vector space $\mathscr{A}^{v}:=\mathscr{A}[[v]]$ of all formal power series in $v$, with coefficients in $\mathscr{A}$. The associative commutative product "." defined on $\mathscr{A}$ is naturally extended to an associative, commutative product on $\mathscr{A}^{v}$, which is still denoted by ".". In the following, any map defined on $\mathscr{A}$ or on $\bigwedge^{\bullet} \mathscr{A}$ is possibly seen as a map on $\mathscr{A}^{v}$ or on $\wedge^{\bullet} \mathscr{A}^{v}$ (the exterior algebra of the $\mathbb{F}^{v}$-vector space $\mathscr{A}^{v}$ ), which means that we consider its natural extension by $\mathbb{F}^{v}$-linearity. We point out that an $\mathbb{F}^{v}-k$-linear map $\psi:\left(\mathscr{A}^{v}\right)^{k} \rightarrow \mathscr{A}^{v}$ can be written as $\psi=\psi_{0}+\psi_{1} v+\cdots+\psi_{n} v^{n}+\cdots$, where each $\psi_{i}$ is a $k$-linear map $\mathscr{A}^{k} \rightarrow \mathscr{A}$. This permits us to write a natural isomorphism $\operatorname{Hom}\left(\left(\mathscr{A}^{v}\right)^{k}, \mathscr{A}^{v}\right) \simeq \operatorname{Hom}\left(\mathscr{A}^{k}, \mathscr{A}\right)[[v]]$ of $\mathbb{F}^{v}$-vector spaces.

A formal deformation of a Poisson structure $\pi_{0}$ on $\mathscr{A}$ is a Poisson structure on the $\mathbb{F}^{v}$-algebra $\mathscr{A}^{v}$ that extends the initial Poisson structure. In other words, it is given by a map $\pi_{*}: \mathscr{A}^{v} \times \mathscr{A}^{v} \rightarrow \mathscr{A}^{v}$ that satisfies the Jacobi identity and is of the form $\pi_{*}=\pi_{0}+\pi_{1} v+\cdots+\pi_{n} v^{n}+\cdots$, where the $\pi_{i}$ are skew-symmetric biderivations of $\mathscr{A}$. If one works modulo $v^{n+1}$ (for $n \in \mathbb{N}$ ), that is, if one replaces 
the $\mathbb{F}^{v}$-algebra $\mathscr{A}^{v}$ with the $\mathbb{F}^{v} /\left\langle v^{n+1}\right\rangle$-algebra $\mathscr{A}^{v} /\left\langle v^{n+1}\right\rangle$ in the previous definition, one then speaks of $n$-th order deformation of $\pi_{0}$.

To have some tools to study formal (or $n$-th order) deformations of Poisson structures, we recall the notion of Poisson cohomology. The Poisson complex is defined as follows: The space of all Poisson cochains is $\mathfrak{X}^{\bullet}(\mathscr{A}):=\bigoplus_{k \in \mathbb{N}} \mathfrak{X}^{k}(\mathscr{A})$, where $\mathfrak{X}^{0}(\mathscr{A})$ is $\mathscr{A}$, and $\mathfrak{X}^{k}(\mathscr{A})$ for all $k \in \mathbb{N}^{*}$ denotes the space of all skew-symmetric $k$-derivations of $\mathscr{A}$, that is, the skew-symmetric $k$-linear maps $\mathscr{A}^{k} \rightarrow \mathscr{A}$ that satisfy the derivation property (5) in each of their arguments. Then the Poisson coboundary operator $\delta_{\pi_{0}}^{k}: \mathfrak{X}^{k}(\mathscr{A}) \rightarrow \mathfrak{X}^{k+1}(\mathscr{A})$ is given by the formula $\delta_{\pi_{0}}^{k}:=-\left[\cdot, \pi_{0}\right]_{S}$, where $[\cdot, \cdot]_{S}: \mathfrak{X}^{p}(\mathscr{A}) \times \mathfrak{X}^{q}(\mathscr{A}) \rightarrow \mathfrak{X}^{p+q-1}(\mathscr{A})$ is the so-called Schouten bracket; see [Laurent-Gengoux et al. $\geq 2008$ ]. The Schouten bracket is a graded Lie bracket that generalizes the commutator of derivations and that is a graded biderivation with respect to the wedge product of multiderivations. It is defined, for $P \in \mathfrak{X}^{p}(\mathscr{A})$, $Q \in \mathfrak{X}^{q}(\mathscr{A})$ and $F_{1}, \ldots, F_{p+q-1} \in \mathscr{A}$, by

$$
\begin{aligned}
& {[P, Q]_{S}\left[F_{1}, \ldots, F_{p+q-1}\right]} \\
& =\sum_{\sigma \in S_{q, p-1}} \epsilon(\sigma) P\left[Q\left[F_{\sigma(1)}, \ldots, F_{\sigma(q)}\right], F_{\sigma(q+1)}, \ldots, F_{\sigma(q+p-1)}\right]- \\
& \quad(-1)^{(p-1)(q-1)} \sum_{\sigma \in S_{p, q-1}} \epsilon(\sigma) Q\left[P\left[F_{\sigma(1)}, \ldots, F_{\sigma(p)}\right], F_{\sigma(p+1)}, \ldots, F_{\sigma(p+q-1)}\right],
\end{aligned}
$$

where $S_{k, \ell}$ for $k, \ell \in \mathbb{N}$ denotes the set of all permutations $\sigma$ of $\{1, \ldots, k+\ell\}$, satisfying $\sigma(1)<\cdots<\sigma(k)$ and $\sigma(k+1)<\cdots<\sigma(k+\ell)$, while $\epsilon(\sigma)$ denotes the signature of such a permutation $\sigma$. Note that, like the case of multilinear maps, it is easy to verify that, for all $k \in \mathbb{N}$, the $\mathbb{F}^{v}$-vector space $\mathfrak{X}^{k}\left(\mathscr{A}^{v}\right)$ of all skew-symmetric $k$-derivations of the associative algebra $\left(\mathscr{A}^{v}, \cdot\right)$ is isomorphic to $\mathfrak{X}^{k}(\mathscr{A})[[v]]$. Indeed, every $\psi \in \mathfrak{X}^{k}\left(\mathscr{A}^{v}\right)$ can be written as $\psi=\psi_{0}+\psi_{1} v+\cdots+\psi_{n} \nu^{n}+\cdots$, where each $\psi_{i}$ is an element of $\mathfrak{X}^{k}(\mathscr{A})$. In the following, the Schouten bracket will often be considered as a map defined on $\mathfrak{X}^{\bullet}\left(\mathscr{A}^{v}\right) \times \mathfrak{X}^{\bullet}\left(\mathscr{A}^{v}\right)$ by extending the original by $\mathbb{F}^{\nu}$-bilinearity. The map thus obtained, which we also denote by $[\cdot, \cdot]_{S}$, is in fact a graded Lie algebra structure on $\mathfrak{X}^{\bullet}\left(\mathscr{A}^{v}\right)$ that could also be defined by a formula analogous to (6).

It is then easy and useful to see that, given a skew-symmetric biderivation $\pi \in \mathfrak{X}^{2}(\mathscr{A})$, the Jacobi identity for $\pi$ is equivalent to the equation $[\pi, \pi]_{S}=0$. Then, because of the graded Jacobi identity satisfied by $[\cdot, \cdot]_{S}$ and the fact that $\left[\pi_{0}, \pi_{0}\right]_{S}=0$, the operator $\delta_{\pi_{0}}$ is a coboundary operator, leading to the Poisson cohomology spaces associated to $\left(\mathscr{A}, \pi_{0}\right)$ and defined by

$$
H^{k}\left(\mathscr{A}, \pi_{0}\right):=\operatorname{Ker} \delta_{\pi_{0}}^{k} / \operatorname{Im} \delta_{\pi_{0}}^{k-1} \quad \text { for } k \in \mathbb{N}^{*}
$$


Elements of $Z^{k}\left(\mathscr{A}, \pi_{0}\right):=\operatorname{Ker} \delta_{\pi_{0}}^{k} \subseteq \mathfrak{X}^{k}(\mathscr{A})$ are the (Poisson) $k$-cocycles, while elements of $B^{k}\left(\mathscr{A}, \pi_{0}\right):=\operatorname{Im} \delta_{\pi_{0}}^{k-1} \subseteq \mathfrak{X}^{k}(\mathscr{A})$ are the (Poisson) $k$-coboundaries.

Also, given a map $\pi_{*}=\pi_{0}+\pi_{1} v+\cdots+\pi_{n} v^{n}+\cdots: \mathscr{A}^{v} \times \mathscr{A}^{v} \rightarrow \mathscr{A}^{v}$, where $\pi_{i} \in \mathfrak{X}^{2}(\mathscr{A})$ is a skew-symmetric biderivation of $\mathscr{A}$ for all $i \in \mathbb{N}$, we have that $\pi_{*}$ is a formal deformation of $\pi_{0}$ if and only if $\left[\pi_{*}, \pi_{*}\right]_{S}=0$, that is, if and only if

$$
\delta_{\pi_{0}}^{2}\left(\pi_{n+1}\right)=\frac{1}{2} \sum_{\substack{i+j=n+1 \\ i, j \geq 1}}\left[\pi_{i}, \pi_{j}\right]_{S}
$$

for all $n \in \mathbb{N}$. Similarly, an $n$-th order deformation $\pi_{(n)}=\pi_{0}+\pi_{1} v+\cdots+\pi_{n} v^{n}$ will extend to an $(n+1)$-st order deformation $\pi_{(n+1)}=\pi_{(n)}+\pi_{n+1} v^{n+1}$ if and only if there exists a $\pi_{n+1} \in \mathfrak{X}^{2}(\mathscr{A})$ that solves (7).

2.2. Equivalent formal deformations. In this subsection, for an arbitrary Poisson algebra $\left(\mathscr{A}, \pi_{0}\right)$ we write a formula, involving only the Schouten bracket $[\cdot, \cdot]_{S}$, for the elements of the equivalence class of a given formal deformation of $\pi_{0}$.

First we recall the notion of equivalence for deformations of $\pi_{0}$. Two formal deformations $\pi_{*}$ and $\pi_{*}^{\prime}$ of $\pi_{0}$ are said to be equivalent if there exists an $\mathbb{F}^{v}$-linear map $\Phi:\left(\mathscr{A}^{v}, \pi_{*}\right) \rightarrow\left(\mathscr{A}^{v}, \pi_{*}^{\prime}\right)$ that is a Poisson morphism and is such that $\Phi$ is the identity modulo $\nu$. In this case we write $\pi_{*} \sim \pi_{*}^{\prime}$, and we call $\Phi$ an equivalence morphism from $\pi_{*}$ to $\pi_{*}^{\prime}$. In other words, an $\mathbb{F}^{v}$-linear map $\Phi: \mathscr{A}^{v} \rightarrow \mathscr{A}^{v}$ is an equivalence morphism from $\pi_{*}$ to $\pi_{*}^{\prime}$ if and only if it is a morphism of associative algebras that is equal to the identity modulo $v$ and satisfies

$$
\Phi\left(\pi_{*}[F, G]\right)=\pi_{*}^{\prime}[\Phi(F), \Phi(G)] \text { for all } F, G \in \mathscr{A}
$$

(and therefore, for all $F, G \in \mathscr{A}^{v}$ ). Of course, if $\Phi$ is an equivalence morphism from $\pi_{*}$ to $\pi_{*}^{\prime}$, then $\Phi^{-1}$ is an equivalence morphism from $\pi_{*}^{\prime}$ to $\pi_{*}$. Similarly, one defines the notion of equivalence for $n$-th order deformations by replacing $\mathbb{F}^{v}$ with $\mathbb{F}^{v} /\left\langle v^{n+1}\right\rangle$ and $\mathscr{A}^{v}$ with $\mathscr{A}^{v} /\left\langle v^{n+1}\right\rangle$.

Now it is straightforward to show that the exponential map gives a bijection between the space $\mathfrak{X}_{0}^{1}\left(\mathscr{A}^{v}\right):=\left\{\xi=\sum_{k \geq 1} \xi_{k} v^{k} \mid \xi_{k} \in \mathfrak{X}^{1}(\mathscr{A}), k \in \mathbb{N}^{*}\right\}$ and the space of all automorphisms of $\mathscr{A}^{v}$ that are equal to the identity modulo $v$. This permits us to write an equivalence morphism $\Phi$ between two formal deformations of $\pi_{0}$ as the image of an element of $\mathfrak{X}_{0}^{1}\left(\mathscr{A}^{v}\right)$ by the exponential map. This implies that the equivalence classes of formal deformations of $\pi_{0}$ can be defined as the equivalence classes of the action, defined as follows, of $\mathfrak{X}_{0}^{1}\left(\mathscr{A}^{v}\right)$ on the formal deformations of $\pi_{0}$. For a formal deformation $\pi_{*}$ of $\pi_{0}$ and for $\xi \in \mathfrak{X}_{0}^{1}\left(\mathscr{A}^{\nu}\right)$, we define this action $\xi \cdot \pi_{*}$ by

$$
\xi \cdot \pi_{*}[F, G]:=e^{\xi}\left(\pi_{*}\left[e^{-\xi}(F), e^{-\xi}(G)\right]\right) \quad \text { for all } F, G \in \mathscr{A} .
$$


It is then possible to show the equality

$$
\xi \cdot \pi_{*}=e^{\operatorname{ad}_{\xi}}\left(\pi_{*}\right), \quad \text { where } \operatorname{ad}_{\xi}:=[\xi, \cdot]_{S} .
$$

This equality involves two notions of exponential,

$$
e^{\xi}:=\sum_{k \in \mathbb{N}} \frac{1}{k !} \xi^{k}: \mathscr{A}^{\mathcal{v}} \rightarrow \mathscr{A}^{\mathcal{v}} \quad \text { and } \quad e^{\operatorname{ad}_{\xi}}:=\sum_{k \in \mathbb{N}} \frac{1}{k !}\left(\operatorname{ad}_{\xi}\right)^{k}: \mathfrak{X}^{\bullet}\left(\mathscr{A}^{\nu}\right) \rightarrow \mathfrak{X}^{\bullet}\left(\mathscr{A}^{\nu}\right),
$$

for $\xi=\xi_{1} v+\xi_{2} v^{2}+\cdots+\xi_{n} v^{n}+\cdots \in \mathfrak{X}_{0}^{1}\left(\mathscr{A}^{v}\right)$, with $\xi_{i} \in \mathfrak{X}^{1}(\mathscr{A})$ for all $i \in \mathbb{N}^{*}$. Here $\operatorname{ad}_{\xi}$ is the graded derivation (of degree 0 ) of the associative algebra $\left(\mathfrak{X}^{\bullet}\left(\mathscr{A}^{v}\right), \wedge\right)$ given by $\operatorname{ad}_{\xi}=[\xi, \cdot]_{S}$. In fact, we have

$$
\begin{aligned}
e^{\xi}\left(\pi_{*}\left[e^{-\xi}(F), e^{-\xi}(G)\right]\right) & \\
& =\pi_{*}[F, G]+\sum_{k \in \mathbb{N}^{*}} \sum_{\substack{r, s, t \in \mathbb{N} \\
r+s+t=k}}(-1)^{s+t} \frac{1}{r ! s ! t !} \xi^{r}\left(\pi_{*}\left[\xi^{s}(F), \xi^{t}(G)\right]\right) \\
& =\pi_{*}[F, G]+\sum_{k \in \mathbb{N}^{*}} \frac{1}{k !}\left(\operatorname{ad}_{\xi}\right)^{k}\left(\pi_{*}\right)[F, G]=e^{\operatorname{ad}_{\xi}}\left(\pi_{*}\right)[F, G],
\end{aligned}
$$

where the second equality is easily proved by induction on $k \in \mathbb{N}^{*}$. This action of $\mathfrak{X}_{0}^{1}\left(\mathscr{A}^{v}\right)$ can be extended on the space $\mathfrak{X}^{\bullet}\left(\mathscr{A}^{v}\right)$ of all skew-symmetric multiderivations of $\mathscr{A}^{v}$, and then the formula $\xi \cdot Q=e^{\operatorname{ad}_{\xi}}(Q)$ still holds for any $Q \in \mathfrak{X}^{\bullet}\left(\mathscr{A}^{v}\right)$.

This result can be seen as an analogue of the well-known formula that links the adjoint representation Ad of a Lie group $\boldsymbol{G}$ on its Lie algebra $\mathfrak{g}$ and the adjoint action ad of $\boldsymbol{G}$ on $\mathfrak{g}: \operatorname{Ad}_{e^{\xi}}=e^{\operatorname{ad}_{\xi}}$ for all $\xi \in \mathfrak{g}$.

Finally, we have obtained the following:

Lemma 2.1. Let $\left(A, \pi_{0}\right)$ be a Poisson algebra. Let $\pi_{*}$ be a formal deformation of $\pi_{0}$. The formal deformations of $\pi_{0}$ that are equivalent to $\pi_{*}$ are precisely the maps $\pi_{*}^{\prime}$ of the form

$$
\pi_{*}^{\prime}=e^{\operatorname{ad}_{\xi}}\left(\pi_{*}\right)=\pi_{*}+\sum_{k \in \mathbb{N}^{*}} \frac{1}{k !} \underbrace{\left[\xi,\left[\xi, \ldots,\left[\xi, \pi_{*}\right]_{S} \ldots\right]_{S}\right]_{S}}_{k \text { brackets }}
$$

with $\xi \in \mathfrak{X}_{0}^{1}\left(\mathscr{A}^{v}\right)$, that is, $\xi=\sum_{k \geq 1} \xi_{k} \nu^{k}$, with $\xi_{k} \in \mathfrak{X}^{1}(\mathscr{A})$ for all $k \in \mathbb{N}^{*}$.

There is an analogous result if one considers instead the formal deformations of an associative commutative or a Lie product, but then the $\xi_{k}$ need not be derivations of $\mathscr{A}$ and the Schouten bracket must be replaced by the corresponding graded Lie algebra structure on the cochains of the Hochschild (Gerstenhaber bracket) or Chevalley-Eilenberg cohomology (Nijenhuis-Richardson bracket). 
2.3. Deformations of Poisson structures in a good case. In this subsection, we prove a proposition which gives, for a certain class of Poisson structures, all formal deformations up to equivalence. Its hypotheses are strong, but we will be able in Section 3 to apply this result to big families of Poisson algebras.

Proposition 2.2. Let $\left(\mathscr{A}, \pi_{0}\right)$ be a Poisson algebra. Suppose that $\left(\vartheta_{k} \in \mathfrak{X}^{2}(\mathscr{A})\right)_{k \in \mathscr{K}}$ is a set of 2-cocycles whose cohomology classes form an $\mathbb{F}$-basis of $H^{2}\left(\mathscr{A}, \pi_{0}\right)$, and define $\mathscr{Y}$ to be the set of all $\boldsymbol{a}=\left(a_{n}^{k} \in \mathbb{F}\right)_{k \in \mathscr{K}, n \in \mathbb{N}^{*}}$ such that the sequence $\left(a_{n_{0}}^{k}\right)_{k \in \mathscr{K}}$ has finite support for every $n_{0} \in \mathbb{N}^{*}$.

Suppose that we have a family $\left(\pi_{*}^{a}\right)_{a \in \mathscr{Y}}$ whose elements are formal deformations of the Poisson structure $\pi_{0}$ and are of the form

$$
\pi_{*}^{a}=\pi_{0}+\sum_{n \in \mathbb{N}^{*}}\left(\Psi_{n}^{a}+\sum_{k \in \mathscr{K}} a_{n}^{k} \vartheta_{k}\right) v^{n}
$$

where $\boldsymbol{a}=\left(a_{n}^{k}\right)_{k \in \mathscr{K}, n \in \mathbb{N}^{*}}$ and, for all $n \in \mathbb{N}^{*}, \Psi_{n}^{a} \in \mathfrak{X}^{2}(\mathscr{A})$ is a skew-symmetric biderivation of $\mathscr{A}$ depending only on the $a_{m}^{k}$ in which $k \in \mathscr{K}$ and $1 \leq m<n$; also $\Psi_{1}^{a}=0$. Then we have the following:

(a) For any formal deformation $\pi_{*}$ of $\pi_{0}$, there exists an element a of $\mathscr{Y}$ such that $\pi_{*}$ is equivalent to $\pi_{*}^{a}$;

(b) For any $m$-th order deformation $\pi_{(m)}$ of $\pi_{0}$ for $m \in \mathbb{N}^{*}$, there exists an element a of $\mathscr{S}$ such that $\pi_{(m)}$ is equivalent to $\pi_{*}^{\boldsymbol{a}}$ modulo $v^{m+1}$, that is, in $\mathscr{A}^{v} /\left\langle v^{m+1}\right\rangle$.

Proof. Let $\pi_{*}=\pi_{0}+\sum_{k \in \mathbb{N}^{*}} \pi_{k} \nu^{k}$ be an arbitrary formal deformation of $\pi_{0}$. According to Lemma 2.1, the existence of an element $\boldsymbol{a}=\left(a_{n}^{k}\right)_{k \in \mathscr{K}, n \in \mathbb{N}^{*}} \in \mathscr{Y}$ such that $\pi_{*} \sim \pi_{*}^{a}$ is equivalent to the existence of an element $\xi=\sum_{k \in \mathbb{N}^{*}} \xi_{k} v^{k} \in \mathfrak{X}_{0}^{1}\left(\mathscr{A}^{v}\right)$ such that $\pi_{*}=e^{\operatorname{ad}_{\xi}}\left(\pi_{*}^{a}\right)$. To simplify the notation, for every $\boldsymbol{a} \in \mathscr{Y}$ and every $\xi \in \mathfrak{X}_{0}^{1}\left(\mathscr{A}^{v}\right)$, we write

$$
\pi_{*}^{a, \xi}:=e^{\operatorname{ad}_{\xi}}\left(\pi_{*}^{a}\right), \quad \pi_{*}^{a, \xi}=\pi_{0}+\sum_{i \in \mathbb{N}^{*}} \pi_{i}^{a, \xi} v^{i}, \quad \pi_{*}^{a}=\pi_{0}+\sum_{i \in \mathbb{N}^{*}} \pi_{i}^{a} v^{i},
$$

with $\pi_{i}^{a}, \xi, \pi_{i}^{a} \in \mathfrak{X}^{2}(\mathscr{A})$ for every $i \in \mathbb{N}^{*}$.

We will then show that, for every $N \in \mathbb{N}^{*}$, there exist $a_{1}^{k}, a_{2}^{k}, \ldots, a_{N}^{k} \in \mathbb{F}$ for $k \in \mathscr{K}$ (such that only a finite number of $a_{i}^{k}$ are nonzero for every $1 \leq i \leq N$ ) and $\xi_{1}, \ldots, \xi_{N} \in \mathfrak{X}^{1}(\mathscr{A})$ such that

$$
\pi_{*}=\pi_{*}^{\boldsymbol{a}_{(N)}, \xi_{(N)}}=e^{\mathrm{ad}_{\xi_{(N)}}\left(\pi_{*}^{\boldsymbol{a}_{(N)}}\right) \bmod v^{N+1} .}
$$

Here $\boldsymbol{a}_{(N)}:=\left(a_{1}^{k}, a_{2}^{k}, \ldots, a_{N}^{k}, 0,0, \ldots\right)_{k \in \mathscr{K}}=\left(b_{n}^{k}\right)_{k \in \mathscr{K}, n \in \mathbb{N}^{*}} \in \mathscr{Y}$, with $b_{n}^{k}=a_{n}^{k}$ for $1 \leq n \leq N$ and $b_{n}^{k}=0$ for $n>N$. Also $\xi_{(N)}:=\xi_{1} v+\cdots+\xi_{N} v^{N} \in \mathfrak{X}_{0}^{1}\left(\mathscr{A}^{v}\right)$. We will proceed by induction on $N \in \mathbb{N}^{*}$. Then to prove part (b), with $m$-th order deformations of $\pi_{0}$, we may use the same proof, but with $N$ in the range $1 \leq N \leq m$. 
First suppose that $N=1$. We know by (7) that $\delta_{\pi_{0}}^{2}\left(\pi_{1}\right)=0$, so that, by definition of the $\vartheta_{k}$, there exist $a_{1}^{k} \in \mathbb{F}$ for all $k \in \mathscr{K}$ (with only a finite number of nonzero $a_{1}^{k}$ ) and an $\xi_{1} \in \mathfrak{X}^{1}(\mathscr{A})$ such that

$$
\pi_{1}=\sum_{k \in \mathscr{K}} a_{1}^{k} \vartheta_{k}-\delta_{\pi_{0}}^{1}\left(\xi_{1}\right)
$$

Writing $\boldsymbol{a}_{(1)}:=\left(a_{1}^{k}, 0,0, \ldots\right)_{k \in \mathscr{K}} \in \mathscr{T}$ and $\xi_{(1)}:=\xi_{1} v \in \mathfrak{X}_{0}^{1}\left(\mathscr{A}^{v}\right)$, we have

$$
\pi_{*}^{\boldsymbol{a}_{(1)}}=\pi_{0}+\sum_{k \in \mathscr{K}} a_{1}^{k} \vartheta_{k} v \bmod v^{2}
$$

and hence the following equalities in $\mathscr{A}^{v} /\left\langle v^{2}\right\rangle$ :

$$
\begin{aligned}
\pi_{*}^{\boldsymbol{a}_{(1)}, \xi_{(1)}} & =e^{\operatorname{ad}_{\xi_{(1)}}\left(\pi_{*}^{\boldsymbol{a}_{(1)}}\right) \bmod v^{2}} \\
& =\pi_{0}+\sum_{k \in \mathscr{H}} a_{1}^{k} \vartheta_{k} v+\left[\xi_{1}, \pi_{0}\right]_{S} v \bmod v^{2} \\
& =\pi_{0}+\pi_{1} v \bmod v^{2} .
\end{aligned}
$$

This achieves the case $N=1$. Suppose now $N \geq 1$, and assume the existence of elements $a_{n}^{k} \in \mathbb{F}$ for $k \in \mathcal{K}$ and $1 \leq n \leq N$ (with only a finite number of nonzero $a_{n_{0}}^{k}$ for every $\left.1 \leq n_{0} \leq N\right)$ and the existence of $\xi_{1}, \ldots, \xi_{N} \in \mathfrak{X}^{1}(\mathscr{A})$ satisfying

$$
\pi_{*}=\pi_{*}^{\boldsymbol{a}_{(N)}, \xi_{(N)}} \bmod v^{N+1},
$$

where

$$
\begin{aligned}
\boldsymbol{a}_{(N)} & :=\left(a_{1}^{k}, a_{2}^{k}, \ldots, a_{N}^{k}, 0,0, \ldots\right)_{k \in \mathscr{K}} \in \mathscr{Y}, \\
\xi_{(N)} & :=\xi_{1} v+\cdots+\xi_{N} v^{N} \in \mathfrak{X}_{0}^{1}\left(\mathscr{A}^{v}\right) .
\end{aligned}
$$

We want to show that this equality can be extended to the rank $N+1$ for some $a_{N+1}^{k} \in \mathbb{F}, k \in \mathscr{K}$ and $\xi_{N+1} \in \mathfrak{X}^{1}(\mathscr{A})$. Since, by induction hypothesis, we have $\pi_{i}=\pi_{i}^{\boldsymbol{a}_{(N)}, \xi_{(N)}}$ for all $1 \leq i \leq N$, Equation (7) implies

$$
\delta_{\pi_{0}}^{2}\left(\pi_{N+1}\right)=\delta_{\pi_{0}}^{2}\left(\pi_{N+1}^{\boldsymbol{a}_{(N)}, \xi_{(N)}}\right),
$$

so that there exist $a_{N+1}^{k} \in \mathbb{F}$ for $k \in \mathscr{K}$ (among which only a finite number are nonzero) and $\xi_{N+1} \in \mathfrak{X}^{1}(\mathscr{A})$ such that

$$
\pi_{N+1}=\pi_{N+1}^{\boldsymbol{a}_{(N)}, \xi_{(N)}}+\sum_{k \in \mathscr{K}} a_{N+1}^{k} \vartheta_{k}-\delta_{\pi_{0}}^{1}\left(\xi_{N+1}\right) .
$$

Proceeding as before, let us write $\boldsymbol{a}_{(N+1)}:=\left(a_{1}^{k}, a_{2}^{k}, \ldots, a_{N+1}^{k}, 0,0, \ldots\right) \in \mathscr{Y}$ and $\xi_{(N+1)}:=\xi_{1} v+\cdots+\xi_{N+1} v^{N+1} \in \mathfrak{X}_{0}^{1}\left(\mathscr{A}^{v}\right)$. By definition of the $\Psi_{n}^{\boldsymbol{b}}$, for $\boldsymbol{b} \in \mathscr{Y}$ and of the elements $\boldsymbol{a}_{(N+1)}$ and $\boldsymbol{a}_{(N)}$, the skew-symmetric biderivation $\Psi_{N+1}^{\boldsymbol{a}_{(N+1)}}$ depends only on the $a_{m}^{k}$ with $k \in \mathscr{K}$ and $1 \leq m<N+1$, that is, only on $\boldsymbol{a}_{(N)}$, and then 
$\Psi_{N+1}^{\boldsymbol{a}_{(N+1)}}=\Psi_{N+1}^{\boldsymbol{a}_{(N)}}$. By definition of the formal deformations of the form $\pi_{*}^{\boldsymbol{b}}$, we then have

$$
\pi_{N+1}^{\boldsymbol{a}_{(N+1)}}=\Psi_{N+1}^{\boldsymbol{a}_{(N)}}+\sum_{k \in \mathscr{K}} a_{N+1}^{k} \vartheta_{k}=\pi_{N+1}^{\boldsymbol{a}_{(N)}}+\sum_{k \in \mathscr{K}} a_{N+1}^{k} \vartheta_{k} .
$$

Then, using the fact that $\pi_{\ell}^{\boldsymbol{a}_{(N+1)}}=\pi_{\ell}^{\boldsymbol{a}_{(N)}}$ for all $\ell<N+1$, we also have

$$
\begin{aligned}
& \pi_{N+1}^{\boldsymbol{a}_{(N+1)}, \xi_{(N+1)}}=\pi_{N+1}^{\boldsymbol{a}_{(N+1)}} \\
& +\sum_{r \in \mathbb{N}^{*}} \frac{1}{r !} \sum_{\ell=0}^{N} \sum_{\substack{i_{1}+\cdots+i_{r}+\ell=N+1 \\
1 \leq i_{1}, \ldots, i_{r} \leq N+1}}\left[\xi_{i_{1}},\left[\xi_{i_{2}}, \ldots,\left[\xi_{i_{r}}, \pi_{\ell}^{\boldsymbol{a}_{(N+1)}}\right]_{S} \cdots\right]_{S}\right]_{S} \\
& =\pi_{N+1}^{\boldsymbol{a}_{(N+1)}}+\left[\xi_{N+1}, \pi_{0}\right]_{S} \\
& +\sum_{r \in \mathbb{N}^{*}} \frac{1}{r !} \sum_{\ell=0}^{N} \sum_{\substack{i_{1}+\cdots+i_{r}+\ell=N+1 \\
1 \leq i_{1}, \ldots, i_{r} \leq N}}\left[\xi_{i_{1}},\left[\xi_{i_{2}}, \ldots,\left[\xi_{i_{r}}, \pi_{\ell}^{\boldsymbol{a}_{(N+1)}}\right]_{S} \cdots\right]_{S}\right]_{S} \\
& =\pi_{N+1}^{\boldsymbol{a}_{(N)}}+\sum_{k \in \mathscr{K}} a_{N+1}^{k} \vartheta_{k}+\left[\xi_{N+1}, \pi_{0}\right]_{S} \\
& +\sum_{r \in \mathbb{N}^{*}} \frac{1}{r !} \sum_{\ell=0}^{N} \sum_{\substack{i_{1}+\cdots+i_{r}+\ell=N+1 \\
1 \leq i_{1}, \ldots, i_{r} \leq N}}\left[\xi_{i_{1}},\left[\xi_{i_{2}}, \ldots,\left[\xi_{i_{r}}, \pi_{\ell}^{\boldsymbol{a}_{(N)}}\right]_{S} \cdots\right]_{S}\right]_{S} \\
& =\pi_{N+1}^{\boldsymbol{a}_{(N)}, \xi_{(N)}}+\sum_{k \in \mathscr{K}} a_{N+1}^{k} \vartheta_{k}+\left[\xi_{N+1}, \pi_{0}\right]_{S}=\pi_{N+1},
\end{aligned}
$$

where, in last step, we used Equation (12). This achieves the proof.

2.4. The case of $\boldsymbol{H}^{\mathbf{1}}\left(\mathscr{A}, \boldsymbol{\pi}_{0}\right)=\{\mathbf{0}\}$. In this subsection, we study equivalent formal deformations of a Poisson structure, under the assumption that the first cohomology space $H^{1}\left(\mathscr{A}, \pi_{0}\right)$ is zero. We will in fact study in Section 3 of this paper a family of Poisson structures for which this space is generically zero. There we will use the result given in this subsection, which first requires the following lemma:

Lemma 2.3. Let $\left(\mathscr{A}, \pi_{0}\right)$ be a Poisson algebra, and let $\pi_{*}$ be a formal deformation of $\pi_{0}$. Suppose that the first Poisson cohomology space of the initial Poisson algebra is zero, that is, $H^{1}\left(\mathscr{A}, \pi_{0}\right)=\{0\}$. Then, we have the following:

(a) The first Poisson cohomology space of the Poisson algebra $\left(\mathscr{A}^{v}, \pi_{*}\right)$ is zero:

$$
H^{1}\left(\mathscr{A}^{v}, \pi_{*}\right)=\{0\} .
$$

(b) For all $N \in \mathbb{N}^{*}$, the first Poisson cohomology space of the Poisson algebra $\left(\mathscr{A}^{v} /\left\langle v^{N}\right\rangle, \pi_{*} \bmod v^{N}\right)$ is zero:

$$
H^{1}\left(\mathscr{A}^{v} /\left\langle v^{N}\right\rangle, \pi_{*} \bmod v^{N}\right)=\{0\} .
$$


Proof. Suppose $\left(\mathscr{A}, \pi_{0}\right)$ is a Poisson algebra such that $H^{1}\left(\mathscr{A}, \pi_{0}\right)=\{0\}$, and let $\pi_{*}=\pi_{0}+\sum_{i \in \mathbb{N}^{*}} \pi_{i} \nu^{i}$ be a formal deformation of $\pi_{0}$. Suppose that $\psi \in \mathfrak{X}^{1}\left(\mathscr{A}^{v}\right)$ is a 1-cocycle for the Poisson algebra $\left(\mathscr{A}^{v}, \pi_{*}\right)$. It means that we have

$$
\left[\psi, \pi_{*}\right]_{S}=0 .
$$

We write $\psi=\sum_{i \in \mathbb{N}} \psi_{i} v^{i}$, with $\psi_{i} \in \mathfrak{X}^{1}(\mathscr{A})$ for all $i \in \mathbb{N}$. Now, to prove part (a), we will show that for all $m \in \mathbb{N}^{*}$, there exist $h_{0}, h_{1}, \ldots, h_{m-1} \in \mathscr{A}$ satisfying

$$
\psi+\left[h_{0}+h_{1} v+\cdots+h_{m-1} v^{m-1}, \pi_{*}\right]_{S}=0 \bmod \nu^{m} .
$$

Indeed, denoting by $H \in \mathscr{A}^{v}$ the element $H=\sum_{i \in \mathbb{N}} h_{i} v^{i}$, this shows that $\psi=$ $-\left[H, \pi_{*}\right]_{S}=\delta_{\pi_{*}}^{1}(H)$ is a 1-coboundary for the Poisson algebra $\left(\mathscr{A}^{v}, \pi_{*}\right)$, and we can conclude that $H^{1}\left(\mathscr{A}^{v}, \pi_{*}\right)=\{0\}$. To prove part (b), it suffices to do the same proof, this time using the range $1 \leq m<N$.

By induction, we will show the equality (14) for all $m \in \mathbb{N}^{*}$. First consider the case $m=1$. In fact, (13) implies in particular that

$$
0=\left[\psi, \pi_{*}\right]_{S} \bmod v=\left[\psi_{0}, \pi_{0}\right]_{S}=-\delta_{\pi_{0}}^{1}\left(\psi_{0}\right) .
$$

Since $H^{1}\left(\mathscr{A}, \pi_{0}\right)=\{0\}$, we then obtain the existence of an element $h_{0} \in \mathscr{A}$ such that $\psi_{0}=\delta_{\pi_{0}}^{0}\left(h_{0}\right)=-\left[h_{0}, \pi_{0}\right]_{S}$, which is exactly (14) for $m=1$.

Now, suppose $m \geq 1$ and that we have $h_{0}, h_{1}, \ldots, h_{m-1} \in \mathscr{A}$ such that

$$
\Psi:=\psi+\left[h_{0}+h_{1} v+\cdots+h_{m-1} v^{m-1}, \pi_{*}\right]_{S} \in \mathfrak{X}^{1}\left(\mathscr{A}^{v}\right)
$$

satisfies $\Psi=0 \bmod v^{m}$. We then write $\Psi=\sum_{i \geq m} \Psi_{i} v^{i}$, with $\Psi_{i} \in \mathfrak{X}^{1}(\mathscr{A})$ for all $i \geq m$. Since $\Psi$ and $\psi$ differ by a 1-coboundary of the Poisson algebra $\left(\mathscr{A}^{v}, \pi_{*}\right)$, Equation (13) together with the fact that $\Psi=0 \bmod v^{m}$ imply that

$$
0=\left[\psi, \pi_{*}\right]_{S} \bmod v^{m+1}=\left[\Psi, \pi_{*}\right]_{S} \bmod v^{m+1}=\left[\Psi_{m}, \pi_{0}\right]_{S} v^{m} .
$$

We then have $\delta_{\pi_{0}}^{1}\left(\Psi_{m}\right)=-\left[\Psi_{m}, \pi_{0}\right]_{S}=0$, and, since $H^{1}\left(\mathscr{A}, \pi_{0}\right)=\{0\}$, there is then an element $h_{m} \in \mathscr{A}$ such that $\Psi_{m}=\delta_{\pi_{0}}^{0}\left(h_{m}\right)$. This can be written as follows:

$$
-\left[h_{m}, \pi_{0}\right]_{S}=\Psi_{m}=\psi_{m}+\sum\left[h_{i}, \pi_{j}\right]_{S},
$$

where the sum is over $i$ and $j$ satisfying $i+j=m, 0 \leq i \leq m-1$ and $j \in \mathbb{N}$. But this is exactly $\psi_{m}=-\sum\left[h_{i}, \pi_{j}\right]_{S}$ over the indices with $i+j=m$ and $i, j \in \mathbb{N}$. Using this and (14), we obtain

$$
\psi+\left[h_{0}+h_{1} v+\cdots+h_{m-1} v^{m-1}+h_{m} v^{m}, \pi_{*}\right]_{S}=0 \bmod v^{m+1} .
$$

Remark 2.4. Lemma 2.3 is also valid if the first Poisson cohomology spaces associated to $\left(A, \pi_{0}\right),\left(\mathscr{A}^{v}, \pi_{*}\right)$ or $\left(\mathscr{A}^{v} /\left\langle v^{N}\right\rangle, \pi_{*} \bmod v^{N}\right)$ are replaced by the $k$-th Poisson cohomology spaces associated to these Poisson algebras. The proof is 
clearly analogous. In fact, in this paper, we will only need the result stated above. In Section 3, we will consider generic Poisson algebras in dimension three, and these will indeed have a first Poisson cohomology space that is zero and nonzero $k$-th Poisson cohomology spaces for $k \in\{0,2,3\}$.

Before the main result of this subsection, let us give another lemma.

Lemma 2.5. Let $\left(\mathcal{A}, \pi_{0}\right)$ be a Poisson algebra. Let us suppose that $\pi_{*} \sim \pi_{*}^{\prime}$ are two equivalent formal deformations of $\pi_{0}$. According to Lemma 2.1, there exists an element $\xi \in \mathfrak{X}_{0}^{1}\left(\mathscr{A}^{v}\right)$ such that $\pi_{*}^{\prime}=e^{\operatorname{ad}_{\xi}}\left(\pi_{*}\right)$. If $\pi_{*}=\pi_{*}^{\prime} \bmod v^{N}$ for some $N \in \mathbb{N}^{*}$, then $\xi \bmod v^{N}$ is a 1-cocycle of the Poisson algebra $\left(\mathscr{A}^{v} /\left\langle v^{N}\right\rangle, \pi_{*} \bmod v^{N}\right)$, that is, $\left[\xi, \pi_{*}\right]_{S} \bmod v^{N}=0$.

Proof. By hypothesis, we have the equality

$$
\pi_{*}^{\prime}=e^{\operatorname{ad}_{\xi}}\left(\pi_{*}\right)=\pi_{*}+\sum_{k \in \mathbb{N}^{*}} \frac{1}{k !} \underbrace{\left[\xi,\left[\xi, \ldots,\left[\xi, \pi_{*}\right]_{S} \cdots\right]_{S}\right]_{S}}_{k \text { brackets }} .
$$

We will prove the desired result by induction on $N \in \mathbb{N}^{*}$. If $N=1$, then the hypothesis becomes the triviality $\pi_{0}=\pi_{0}$, and $\xi \bmod v=0$ is trivially a 1-cocycle of the Poisson algebra $\left(\mathscr{A}, \pi_{0}\right)$.

Now, let $N \geq 1$ and suppose that if $\pi_{*}=\pi_{*}^{\prime} \bmod v^{N}$, then $\xi \bmod v^{N}$ is a 1-cocycle of the Poisson algebra $\left(\mathscr{A}^{v} /\left\langle v^{N}\right\rangle, \pi_{*} \bmod v^{N}\right)$. Under the assumption that $\pi_{*}=\pi_{*}^{\prime} \bmod v^{N+1}$, we have $\pi_{*}=\pi_{*}^{\prime} \bmod v^{N}$ and, by induction hypothesis, $\left[\xi, \pi_{*}\right]_{S} \bmod v^{N}=0$. This last equality and Equation (16) imply

$$
0=\sum_{k \in \mathbb{N}^{*}} \frac{1}{k !} \overbrace{\left[\xi,\left[\xi, \ldots,\left[\xi, \pi_{*}\right]_{S} \cdots\right]_{S}\right]_{S}}^{k \text { brackets }} \bmod v^{N+1}=\left[\xi, \pi_{*}\right]_{S} \bmod v^{N+1},
$$

which exactly implies that $\xi \bmod v^{N+1}$ is a Poisson 1-cocycle of the Poisson algebra $\left(\mathscr{A}^{v} /\left\langle v^{N+1}\right\rangle, \pi_{*} \bmod v^{N+1}\right)$.

Now let us give the main result of this subsection.

Proposition 2.6. Let $\left(\mathscr{A}, \pi_{0}\right)$ be a Poisson algebra whose first Poisson cohomology space is zero, that is, $H^{1}\left(\mathscr{A}, \pi_{0}\right)=\{0\}$. Let us suppose that $\pi_{*}=\pi_{0}+\sum_{i \in \mathbb{N}^{*}} \pi_{i} v^{i}$ and $\pi_{*}^{\prime}=\pi_{0}+\sum_{i \in \mathbb{N}^{*}} \pi_{i}^{\prime} v^{i}$ (with $\pi_{i}, \pi_{i}^{\prime} \in \mathfrak{X}^{2}(\mathscr{A})$ for $i \in \mathbb{N}^{*}$ ) are two equivalent formal deformations of $\pi_{0}$. If $\pi_{*}=\pi_{*}^{\prime} \bmod v^{N}$ for some $N \in \mathbb{N}^{*}$, then there exists $a \psi \in \mathfrak{X}^{1}(\mathscr{A})$ such that $\pi_{N}-\pi_{N}^{\prime}=\delta_{\pi_{0}}^{1}(\psi)$.

Proof. Let us consider a Poisson algebra $\left(\mathscr{A}, \pi_{0}\right)$. We suppose that $\pi_{*}$ and $\pi_{*}^{\prime}$ are two equivalent formal deformations of $\pi_{0}$. According to Lemma 2.1, there exists a $\xi=\sum_{k \in \mathbb{N}^{*}} \xi_{k} \nu^{k} \in \mathfrak{X}_{0}^{1}\left(\mathscr{A}^{\nu}\right)$ satisfying $\pi_{*}^{\prime}=e^{\operatorname{ad} \xi}\left(\pi_{*}\right)$. Assume that $\pi_{*}=\pi_{*}^{\prime} \bmod v^{N}$ for some $N \in \mathbb{N}^{*}$. Then Lemma 2.5 implies that $\xi \bmod \nu^{N}$ is a 1-cocycle of the Poisson algebra $\left(\mathscr{A}^{v} /\left\langle v^{N}\right\rangle, \pi_{*} \bmod v^{N}\right)$. By hypothesis, $H^{1}\left(\mathscr{A}, \pi_{0}\right)=\{0\}$, 
so that, according to Lemma 2.3(b), there exists an element $H \in \mathscr{A}^{v}$ such that $\mathscr{X}:=\xi+\left[H, \pi_{*}\right]_{S} \in \mathfrak{X}^{1}\left(\mathscr{A}^{v}\right)$ satisfies $\mathscr{X}=0 \bmod v^{N}$. We then write $\mathscr{X}=\sum_{i \geq N} \mathscr{X}_{i} v^{i}$, with $\mathscr{X}_{i} \in \mathfrak{X}^{1}(\mathscr{A})$ for all $i \geq N$. Now, because

$\xi \bmod v^{N+1}=-\left[H, \pi_{*}\right]_{S}+\mathscr{X}_{N} v^{N} \bmod v^{N+1}$ and $\left[\left[H, \pi_{*}\right]_{S}, \pi_{*}\right]_{S}=0$,

we have

$$
\pi_{*}-\pi_{*}^{\prime} \bmod v^{N+1}=\pi_{*}-e^{\operatorname{ad}_{\xi}}\left(\pi_{*}\right) \bmod v^{N+1}=-\left[\mathscr{Q}_{N}, \pi_{0}\right]_{S} v^{N} .
$$

We conclude that $\pi_{N}-\pi_{N}^{\prime}=-\left[\mathscr{X}_{N}, \pi_{0}\right]_{S}=\delta_{\pi_{0}}^{1}\left(\mathscr{X}_{N}\right)$ with $\mathscr{X}_{N} \in \mathfrak{X}^{1}(\mathscr{A})$, as desired.

Combining Propositions 2.2 and 2.6 gives Proposition 1.1. In particular, we obtain this:

Proposition 2.7. Let $\left(\mathscr{A}, \pi_{0}\right)$ be a Poisson algebra. With the notation and hypotheses of Proposition 2.2 and with the additional hypothesis that the first Poisson cohomology space $H^{1}\left(\mathcal{A}, \pi_{0}\right)$ is zero, we have the following:

(a) For any formal deformation $\pi_{*}$ of $\pi_{0}$, there exists a unique element a of $\mathscr{S}$ such that $\pi_{*}$ is equivalent to $\pi_{*}^{a}$.

(b) For any $m$-th order deformation $\pi_{(m)}$ of $\pi_{0}$ (with $\left.m \in \mathbb{N}^{*}\right)$, there exists a unique element $\boldsymbol{a}_{(m+1)} \in \mathscr{S}$ of the form $\boldsymbol{a}_{(m+1)}=\left(a_{1}^{k}, a_{2}^{k}, \ldots, a_{m}^{k}, 0,0, \ldots\right)_{k \in \mathscr{K}}$ (that $i s, \boldsymbol{a}_{(m+1)}=\left(a_{n}^{k}\right)_{k \in \mathscr{K}, n \in \mathbb{N}^{*}}$ with $a_{n}^{k}=0$ for every $k \in \mathscr{K}$ and $\left.n \geq m+1\right)$ such that $\pi_{(m)}$ is equivalent to $\pi_{*}^{\boldsymbol{a}_{(m+1)}}$ modulo $v^{m+1}$, that is, in $\mathscr{A}^{v} /\left\langle v^{m+1}\right\rangle$.

Proof. The existence of the elements $\boldsymbol{a}$ and $\boldsymbol{a}_{(m+1)}$ are given by Proposition 2.2, so we now study the uniqueness. To do this, we point out that if $\boldsymbol{a}=\left(a_{n}^{k}\right)_{k \in \mathscr{K}, n \in \mathbb{N}^{*}}$ and $\boldsymbol{b}=\left(b_{n}^{k}\right)_{k \in \mathscr{K}, n \in \mathbb{N}^{*}}$ are elements of $\mathscr{Y}$ defining two different formal deformations $\pi_{*}^{a}$ and $\pi_{*}^{b}$ of the form (9) and if $N:=\min \left\{n \in \mathbb{N}^{*} \mid \pi_{n}^{a} \neq \pi_{n}^{\boldsymbol{b}}\right\}$, then $\Psi_{N}^{\boldsymbol{a}}=\Psi_{N}^{\boldsymbol{b}}$ and $\pi_{N}^{a}-\pi_{N}^{b}$ is an element of $\bigoplus_{k \in \mathscr{K}} \mathbb{F} \vartheta_{k}$ that is complementary to $B^{2}\left(\mathscr{A}, \pi_{0}\right)$ in $Z^{2}\left(\mathscr{A}, \pi_{0}\right)$. According to Proposition 2.6, if $\pi_{*}^{a}$ and $\pi_{*}^{b}$ were equivalent, then $\pi_{N}^{a}-\pi_{N}^{b}$ would be a Poisson coboundary of $\left(\mathscr{A}, \pi_{0}\right)$ (an element of $B^{2}\left(\mathscr{A}, \pi_{0}\right)$ ); we then conclude that $\pi_{*}^{a}$ and $\pi_{*}^{b}$ cannot be equivalent.

Remark 2.8. This result, and also Propositions 2.2 and 2.6, could be stated in an associative or Lie context in a very analogous way (by replacing the Poisson cohomology by the Hochschild or Chevalley-Eilenberg cohomology and the Schouten bracket by the appropriate graded Lie algebra structure on the spaces of cochains).

\section{Formal deformations of Poisson structures in dimension two and three}

In this section, we consider a large family of Poisson structures on the affine space of dimension three $\mathbb{F}^{3}$ and on singular surfaces in $\mathbb{F}^{3}$. We study their formal deformations. Using the general results obtained in Section 2 and the Poisson 
cohomology of these Poisson structures obtained in [Pichereau 2006], we find an explicit expression of all their formal deformations, up to equivalence. For more details about these Poisson brackets and their Poisson cohomology, see [Pichereau 2006]. As before, $\mathbb{F}$ denotes an arbitrary field of characteristic zero.

3.1. Poisson structures on $\mathbb{F}^{3}$ associated to a polynomial. In this subsection, we denote by $\mathscr{A}$ the polynomial algebra $\mathscr{A}=\mathbb{F}[x, y, z]$. To each polynomial $\varphi \in \mathscr{A}$, one associates naturally a Poisson structure $\{\cdot, \cdot\}_{\varphi}$ on $\mathscr{A}$ defined by the brackets

$$
\{x, y\}_{\varphi}=\frac{\partial \varphi}{\partial z}, \quad\{y, z\}_{\varphi}=\frac{\partial \varphi}{\partial x}, \quad\{z, x\}_{\varphi}=\frac{\partial \varphi}{\partial y} .
$$

It is indeed easy to show that the skew-symmetric biderivation $\{\cdot, \cdot\}_{\varphi}$ explicitly given by

$$
\{\cdot, \cdot\}_{\varphi}=\frac{\partial \varphi}{\partial z} \frac{\partial}{\partial x} \wedge \frac{\partial}{\partial y}+\frac{\partial \varphi}{\partial x} \frac{\partial}{\partial y} \wedge \frac{\partial}{\partial z}+\frac{\partial \varphi}{\partial y} \frac{\partial}{\partial z} \wedge \frac{\partial}{\partial x}
$$

satisfies the Jacobi identity, that is, it equips the associative commutative algebra $\mathscr{A}$ with a Poisson structure. In the following, we will assume that $\varphi$ is a weighthomogeneous polynomial of (weighted) degree $\varpi(\varphi) \in \mathbb{N}$, that is, that there exist (unique) coprime positive integers $\varpi_{1}, \varpi_{2}, \varpi_{3} \in \mathbb{N}^{*}$ (the weights of the variables $x, y$ and $z$ ) such that

$$
\varpi_{1} x \frac{\partial \varphi}{\partial x}+\varpi_{2} y \frac{\partial \varphi}{\partial y}+\varpi_{3} z \frac{\partial \varphi}{\partial z}=\varpi(\varphi) \varphi .
$$

This equation is also called the Euler formula. For such a weight-homogeneous polynomial $\varphi$ with a so-called isolated singularity (at the origin), the Poisson cohomology of the Poisson algebra $\left(\mathscr{A},\{\cdot, \cdot\}_{\varphi}\right)$ was explicitly determined in [Pichereau 2006]. Recall that a weight-homogeneous polynomial $\varphi \in \mathbb{F}[x, y, z]$ is said to have an isolated singularity (at the origin) if the vector space

$$
\mathscr{A}_{\text {sing }}(\varphi):=\mathbb{F}[x, y, z] /\left\langle\frac{\partial \varphi}{\partial x}, \frac{\partial \varphi}{\partial y}, \frac{\partial \varphi}{\partial z}\right\rangle
$$

is finite-dimensional. Its dimension is then denoted by $\mu$ and called the Milnor number associated to $\varphi$. When $\mathbb{F}=\mathbb{C}$, this amounts, geometrically, to saying that the surface $\mathscr{F}_{\varphi}:\{\varphi=0\}$ has a singular point only at the origin. In [Pichereau 2006], we showed that $\mathscr{A}_{\text {sing }}(\varphi)$, and hence the singularity of $\varphi$, intervenes in the Poisson cohomology of $\left(\mathscr{A},\{\cdot, \cdot\}_{\varphi}\right)$. In the following, we will see that it also appears in the formal deformations of $\{\cdot, \cdot\}_{\varphi}$.

In the following, the polynomial $\varphi$ will always be a weight-homogeneous polynomial with an isolated singularity. The corresponding weights of the three variables $\left(\varpi_{1}, \varpi_{2}\right.$ and $\left.\varpi_{3}\right)$ are then fixed, and the weight-homogeneity of any polynomial in $\mathbb{F}[x, y, z]$ must now be understood with respect to these weights. We will 
also use the fact that, for $\mathscr{A}=\mathbb{F}[x, y, z]$, we have natural isomorphisms

$$
\mathfrak{X}^{0}(\mathscr{A}) \simeq \mathfrak{X}^{3}(\mathscr{A}) \simeq \mathscr{A}, \quad \mathfrak{X}^{1}(\mathscr{A}) \simeq \mathfrak{X}^{2}(\mathscr{A}) \simeq \mathscr{A}^{3},
$$

chosen as

$$
\begin{aligned}
\mathfrak{X}^{1}(\mathscr{A}) \rightarrow \mathscr{A}^{3}, & V \mapsto(V[x], V[y], V[z]), \\
\mathfrak{X}^{2}(\mathscr{A}) \rightarrow \mathscr{A}^{3}, & V \mapsto(V[y, z], V[z, x], V[x, y]), \\
\mathfrak{X}^{3}(\mathscr{A}) \rightarrow \mathscr{A}, & V \mapsto V[x, y, z] .
\end{aligned}
$$

The elements of $\mathscr{A}^{3}$ are viewed as vector-valued functions on $\mathscr{A}$, so we denote them with an arrow, like $\vec{F} \in \mathscr{A}^{3}$. In $\mathscr{A}^{3}$, let $\cdot$ and $\times$ denote respectively the usual inner and cross products, and let $\vec{\nabla}, \vec{\nabla} \times$ and Div denote respectively the gradient, curl and divergence operators. For example, with these notations and the above isomorphisms, the skew-symmetric biderivation $\{\cdot, \cdot\}_{\varphi}$ is identified with the element $\vec{\nabla} \varphi$ of $\mathscr{A}^{3}$. Similarly, the so-called Euler derivation (associated to the weights of the variables),

$$
\vec{e}_{\varpi}:=\varpi_{1} x \frac{\partial}{\partial x}+\varpi_{2} y \frac{\partial}{\partial y}+\varpi_{3} z \frac{\partial}{\partial z}
$$

is viewed as the element $\vec{e}_{\varpi}:=\left(\varpi_{1} x, \varpi_{2} y, \varpi_{3} z\right) \in \mathscr{A}^{3}$, and with the notations above, the Euler formula (19) for a weight-homogeneous polynomial $F \in \mathscr{A}$ of (weighted) degree $\varpi(F)$ becomes

$$
\vec{\nabla} F \cdot \vec{e}_{\varpi}=\varpi(F) F
$$

Remark 3.1. Using the identifications above, it is possible to write the Poisson coboundary operator associated to $\left(\mathscr{A},\{\cdot, \cdot\}_{\varphi}\right)$ in terms of elements in $\mathscr{A}$ and elements in $\mathscr{A}^{3}$. Denoting this coboundary operator by $\delta_{\varphi}^{k}$, we obtain

$$
\begin{array}{ll}
\delta_{\varphi}^{0}(F)=\vec{\nabla} F \times \vec{\nabla} \varphi & \text { for } F \in \mathscr{A} \simeq \mathfrak{X}^{0}(\mathscr{A}), \\
\delta_{\varphi}^{1}(\vec{F})=-\vec{\nabla}(\vec{F} \cdot \vec{\nabla} \varphi)+\operatorname{Div}(\vec{F}) \vec{\nabla} \varphi & \text { for } \vec{F} \in \mathscr{A}^{3} \simeq \mathfrak{X}^{1}(\mathscr{A}), \\
\delta_{\varphi}^{2}(\vec{F})=-\vec{\nabla} \varphi \cdot(\vec{\nabla} \times \vec{F}) & \text { for } \vec{F} \in \mathscr{A}^{3} \simeq \mathfrak{X}^{2}(\mathscr{A}) .
\end{array}
$$

From [Pichereau 2006], we know that if $\varphi$ is a weight-homogeneous polynomial with an isolated singularity, then the Casimirs of the Poisson algebra $\left(\mathscr{A},\{\cdot, \cdot\}_{\varphi}\right)$ (that is, the elements of the center of the Poisson bracket, which are also the elements of $\left.H^{0}\left(\mathscr{A},\{\cdot, \cdot\}_{\varphi}\right)=\operatorname{Ker} \delta_{\varphi}^{0}\right)$ are exactly the polynomials in $\varphi$.

3.2. The second Poisson cohomology space of $\left(\mathscr{A},\{\cdot, \cdot\}_{\varphi}\right)$. From [Pichereau 2006] we recall that, since $\varphi \in \mathbb{F}[x, y, z]$ is a weight-homogeneous polynomial with an isolated singularity, the second Poisson cohomology space associated to 
$\left(\mathscr{A},\{\cdot, \cdot\}_{\varphi}\right)$ is given by

$$
\begin{aligned}
& H^{2}\left(\mathscr{A},\{\cdot, \cdot\}_{\varphi}\right) \simeq \\
& \bigoplus_{\substack{j=1 \\
\varpi\left(u_{j}\right) \neq \omega(\varphi)-|\varpi|}}^{\mu-1} \mathbb{F}[\varphi] \vec{\nabla} u_{j} \oplus \bigoplus_{\substack{j=0 \\
\sigma\left(u_{j}\right)=\varpi(\varphi)-|\varpi|}}^{\mu-1} \mathbb{F}[\varphi] u_{j} \vec{\nabla} \varphi \oplus \bigoplus_{\substack{j=1 \\
\sigma\left(u_{j}\right)=\varpi(\varphi)-|\varpi|}}^{\mu-1} \mathbb{F} \vec{\nabla} u_{j},
\end{aligned}
$$

where $|\varpi|:=\varpi_{1}+\varpi_{2}+\varpi_{3}$ denotes the sum of the weights of the three variables and where the family $u_{0}:=1, u_{1}, \ldots, u_{\mu-1} \in \mathscr{A}$ is composed of weighthomogeneous polynomials in $\mathscr{A}$ whose images in $\mathscr{A}_{\text {sing }}(\varphi)$ give a basis of this $\mathbb{F}$-vector space. To study the formal deformations of the Poisson bracket $\{\cdot, \cdot\}_{\varphi}$, we need another basis of $H^{2}(\mathscr{A}, \varphi)$.

Lemma 3.2. If $\varphi \in \mathscr{A}=\mathbb{F}[x, y, z]$ is a weight-homogeneous polynomial with an isolated singularity, then the second Poisson cohomology space associated to $\left(\mathscr{A},\{\cdot, \cdot\}_{\varphi}\right)$ is the $\mathbb{E}[\varphi]$-module

$$
\begin{aligned}
H^{2}\left(A,\{\cdot, \cdot\}_{\varphi}\right) & \simeq \begin{cases}\bigoplus_{j=0}^{\mu-1} \mathbb{F}[\varphi] u_{j} \vec{\nabla} \varphi \oplus \bigoplus_{j=1}^{\mu-1} \mathbb{F} \vec{\nabla} u_{j} & \text { if } \varpi(\varphi)=|\varpi|, \\
\bigoplus_{j=1}^{\mu-1} \mathbb{F}[\varphi] u_{j} \vec{\nabla} \varphi \oplus \bigoplus_{j=1}^{\mu-1} \mathbb{F} \vec{\nabla} u_{j} \quad \text { if } \varpi(\varphi) \neq|\varpi|,\end{cases} \\
& \simeq \bigoplus_{j \in \mathscr{E}_{\varphi}} \mathbb{F}[\varphi] u_{j} \vec{\nabla} \varphi \oplus \bigoplus_{j=1}^{\mu-1} \mathbb{F} \vec{\nabla} u_{j},
\end{aligned}
$$

where we have used the above notation and where we have defined

$$
\mathscr{E}_{\varphi}:= \begin{cases}\{0, \ldots, \mu-1\} & \text { if } \varpi(\varphi)=|\varpi| \\ \{1, \ldots, \mu-1\} & \text { if } \varpi(\varphi) \neq|\varpi| .\end{cases}
$$

Proof. Using (23), we can compute, for all $i \in \mathbb{N}$ and $0 \leq j \leq \mu-1$,

$$
\delta_{\varphi}^{1}\left(\varphi^{i} u_{j} \vec{e}_{\varpi}\right)=\left(\varpi\left(u_{j}\right)-\varpi(\varphi)+|\varpi|\right) \varphi^{i} u_{j} \vec{\nabla} \varphi-\varpi(\varphi) \varphi^{i+1} \vec{\nabla} u_{j} .
$$

Now, using this equation, it is easy to verify that (24) can also be written as (25).

3.3. The formal deformations of $\{\cdot, \cdot\}_{\varphi}$. We now consider the formal deformations of the Poisson bracket $\{\cdot, \cdot\}_{\varphi}$ on $\mathbb{F}^{3}$, where $\varphi$ is a weight-homogeneous polynomial with an isolated singularity. For this work, the Poisson cohomology that appears is the one associated to the Poisson algebra $\left(\mathscr{A}=\mathbb{F}[x, y, z],\{\cdot, \cdot\}_{\varphi}\right)$.

We first need to obtain a formula for the Schouten bracket of two specific skewsymmetric biderivations of $\mathscr{A}$. In fact, for the study of the formal deformations of $\{\cdot, \cdot\}_{\varphi}$, we will see that we need only consider the skew-symmetric biderivations 
of the form $F \vec{\nabla} G \in \mathscr{A}^{3} \simeq \mathfrak{X}^{2}(\mathscr{A})$, with $F, G \in \mathscr{A}$. Let us compute the Schouten bracket of two such skew-symmetric biderivations. So let $F, G, H, L \in \mathscr{A}$. We compute the Schouten bracket $[F \vec{\nabla} L, G \vec{\nabla} H]_{S} \in \mathfrak{X}^{3}(\mathscr{A}) \simeq \mathscr{A}$, which we identify (according to (21)) with its value $[F \vec{\nabla} L, G \vec{\nabla} H]_{S}[x, y, z] \in \mathscr{A}$, and obtain

$$
[F \vec{\nabla} L, G \vec{\nabla} H]_{S}=F \vec{\nabla} L \cdot(\vec{\nabla} G \times \vec{\nabla} H)+G \vec{\nabla} H \cdot(\vec{\nabla} F \times \vec{\nabla} L) .
$$

According to this equation, we have, for every $l, m \in \mathbb{N}$ and every $0 \leq i, j \leq \mu-1$,

$$
\left[\varphi^{l} u_{i} \vec{\nabla} \varphi, \varphi^{m} u_{j} \vec{\nabla} \varphi\right]_{S}=0 \quad \text { and } \quad\left[\vec{\nabla} u_{i}, \vec{\nabla} u_{j}\right]_{S}=0,
$$

while, with the help of (26) and (23), we obtain

$$
\left[\varphi^{l} u_{i} \vec{\nabla} \varphi, \vec{\nabla} u_{j}\right]_{S}=\delta_{\varphi}^{2}\left(\varphi^{l} u_{i} \vec{\nabla} u_{j}\right)
$$

The following proposition gives a formula for all formal deformations of $\{\cdot, \cdot\}_{\varphi}$, up to equivalence.

Proposition 3.3. Let $\varphi \in \mathscr{A}=\mathbb{F}[x, y, z]$ be a weight-homogeneous polynomial with an isolated singularity. Consider the Poisson algebra $\left(\mathscr{A},\{\cdot, \cdot\}_{\varphi}\right)$ associated to $\varphi$, where $\pi_{0}:=\{\cdot, \cdot\}_{\varphi}$ is the Poisson bracket given by

$$
\{\cdot, \cdot\}_{\varphi}=\frac{\partial \varphi}{\partial x} \frac{\partial}{\partial y} \wedge \frac{\partial}{\partial z}+\frac{\partial \varphi}{\partial y} \frac{\partial}{\partial z} \wedge \frac{\partial}{\partial x}+\frac{\partial \varphi}{\partial z} \frac{\partial}{\partial x} \wedge \frac{\partial}{\partial y} .
$$

Then we have the following:

(a) For all families of constants

$$
\left(c_{l, i}^{k} \in \mathbb{F}\right)_{(l, i) \in \mathbb{N} \times \mathscr{E}_{\varphi}, k \in \mathbb{N}^{*}} \quad \text { and } \quad\left(\bar{c}_{r}^{k} \in \mathbb{F}\right)_{1 \leq r \leq \mu-1, k \in \mathbb{N}^{*}}
$$

such that, for every $k_{0} \in \mathbb{N}^{*}$, the sequences $\left(c_{l, i}^{k_{0}}\right)_{(l, i) \in \mathbb{N} \times \mathscr{E}_{\varphi}}$ and $\left(\bar{c}_{r}^{k_{0}}\right)_{1 \leq r \leq \mu-1}$ have finite support, the formula

$$
\begin{aligned}
& \pi_{*}=\{\cdot, \cdot\}_{\varphi}+\sum_{n \in \mathbb{N}^{*}} \pi_{n} v^{n}, \quad \text { where } \\
& \pi_{n}=\sum_{\substack{(l, i) \in \mathbb{N} \times \mathscr{E}_{\varphi} \\
1 \leq r \leq \mu-1}} \sum_{\substack{a+b=n \\
a, b \in \mathbb{N}^{*}}} c_{l, i}^{a} \bar{c}_{r}^{b} \varphi^{l} u_{i} \vec{\nabla} u_{r} \\
& +\sum_{(m, j) \in \mathbb{N} \times \mathscr{E}_{\varphi}} c_{m, j}^{n} \varphi^{m} u_{j} \vec{\nabla} \varphi+\sum_{1 \leq s \leq \mu-1} \bar{c}_{s}^{n} \vec{\nabla} u_{s},
\end{aligned}
$$

defines a formal deformation of $\{\cdot, \cdot\}_{\varphi}$, where the $u_{j}$ for $0 \leq j \leq \mu-1$ are weight-homogeneous polynomials of $\mathscr{A}=\mathbb{F}[x, y, z]$ whose images in

$$
\mathscr{A}_{\text {sing }}(\varphi)=\mathbb{F}[x, y, z] /\left\langle\frac{\partial \varphi}{\partial x}, \frac{\partial \varphi}{\partial y}, \frac{\partial \varphi}{\partial z}\right\rangle
$$

give a basis of the $\mathbb{F}$-vector space $A_{\text {sing }}(\varphi)\left(\right.$ and $\left.u_{0}=1\right)$. 
(b) For any formal deformation $\pi_{*}^{\prime}$ of $\{\cdot, \cdot\}_{\varphi}$, there exist families of constants

$$
\left(c_{l, i}^{k}\right)_{(l, i) \in \mathbb{N} \times \mathscr{E}_{\varphi}, k \in \mathbb{N}^{*}} \text { and }\left(\bar{c}_{r}^{k}\right)_{1 \leq r \leq \mu-1, k \in \mathbb{N}^{*}}
$$

(such that only a finite number of $c_{l, i}^{k_{0}}$ and $\bar{c}_{r}^{k_{0}}$ are nonzero for every $k_{0} \in \mathbb{N}^{*}$ ) for which $\pi_{*}^{\prime}$ is equivalent to the formal deformation $\pi_{*}$ given by (29).

(c) Moreover, if the (weighted) degree of the polynomial $\varphi$ is not equal to the sum of the weights $\varpi(\varphi) \neq|\varpi|$, then for any formal deformation $\pi_{*}^{\prime}$ of $\{\cdot, \cdot\}_{\varphi}$, there exist unique families of constants

$$
\left(c_{l, i}^{k}\right)_{(l, i) \in \mathbb{N} \times \mathscr{E}_{\varphi}, k \in \mathbb{N}^{*}} \text { and }\left(\bar{c}_{r}^{k}\right)_{1 \leq r \leq \mu-1, k \in \mathbb{N}^{*}}
$$

(with only a finite number of nonzero $c_{l, i}^{k_{0}}$ and $\bar{c}_{r}^{k_{0}}$ for every $k_{0} \in \mathbb{N}^{*}$ ) such that $\pi_{*}^{\prime}$ is equivalent to the formal deformation $\pi_{*}$ given by (29).

This means that (29) gives a system of representatives for all formal deformations of $\{\cdot, \cdot\}_{\varphi}$, modulo equivalence.

(d) Analogous results hold if we replace formal deformations by $m$-th order deformations (with $m \in \mathbb{N}^{*}$ ) and impose in (c) that $c_{l, i}^{k}=0$ and $\bar{c}_{r}^{k}=0$ for $k \geq m+1$.

Remark 3.4. In particular, the previous proposition implies that, if $\varpi(\varphi) \neq|\varpi|$, the formal deformations of $\{\cdot, \cdot\}_{\varphi}$ defined by (29) and different from $\{\cdot, \cdot\}_{\varphi}$ (that is, the constants $c_{l, i}^{k} \in \mathbb{F}$ and $\bar{c}_{r}^{k} \in \mathbb{F}$ are not all zero) are all nontrivial formal deformations of $\{\cdot, \cdot\}_{\varphi}$ (that is, they are not equivalent to $\{\cdot, \cdot\}_{\varphi}$ ).

Proof. In fact, by proving the part (a), we will show that the Poisson algebra $\left(\mathscr{A},\{\cdot, \cdot\}_{\varphi}\right)$ verifies the hypotheses of Proposition 2.2, with

$$
\begin{aligned}
\mathscr{K} & =\left(\mathbb{N} \times \mathscr{E}_{\varphi}\right) \cup\{1, \ldots, \mu-1\}, \\
\boldsymbol{a} & =\left(c_{l, i}^{a}, \bar{c}_{r}^{b} \mid(l, i) \in \mathbb{N} \times \mathscr{E}_{\varphi}, 1 \leq r \leq \mu-1,1 \leq a, b \leq n\right)_{n \in \mathbb{N}^{*} \in \mathscr{Y},} \\
\vartheta_{r, j} & =\varphi^{r} u_{j} \vec{\nabla} \varphi \quad \text { for }(r, j) \in \mathbb{N} \times \mathscr{E}_{\varphi}, \\
\vartheta_{i} & =\vec{\nabla} u_{i} \quad \text { for } 1 \leq i \leq \mu-1, \\
\Psi_{n}^{a} & =\sum_{\substack{(l, i) \in \mathbb{N} \times \mathscr{E}_{\varphi} \\
r \in\{1, \ldots, \mu-1\}}} \sum_{a+b=n} c_{l, i}^{a} \bar{c}_{r}^{b} \varphi^{l} u_{i} \vec{\nabla} u_{r},
\end{aligned}
$$

which implies part (b). According to Lemma 3.2, the elements $\varphi^{r} u_{j} \vec{\nabla} \varphi$ and $\vec{\nabla} u_{i}$ for $(r, j) \in \mathbb{N} \times \mathscr{E}_{\varphi}$ and $1 \leq i \leq \mu-1$ give an $\mathbb{F}$-basis of the second Poisson cohomology space $H^{2}\left(\mathscr{A},\{\cdot, \cdot\}_{\varphi}\right)$, so it suffices, for (a) and (b), to show that (29) defines a formal deformation of $\pi_{0}=\{\cdot, \cdot\}_{\varphi}$. Let us consider some constants $c_{l, i}^{k} \in \mathbb{F}$ and $\bar{c}_{r}^{k} \in \mathbb{F}$, with $(l, i) \in \mathbb{N} \times \mathscr{E}_{\varphi}, 1 \leq r \leq \mu-1$ and $k \in \mathbb{N}^{*}$, and consider 
$\pi_{*}=\{\cdot, \cdot\}_{\varphi}+\sum_{k \in \mathbb{N}^{*}} \pi_{k} v^{k}$ with

$$
\begin{aligned}
\pi_{k}= & \sum_{\substack{(l, i) \in \mathbb{N} \times \mathscr{E}_{\varphi} \\
r \in\{1, \ldots, \mu-1\}}} \sum_{\substack{a+b=k \\
a, b \in \mathbb{N}^{*}}} c_{l, i}^{a} \bar{c}_{r}^{b} \varphi^{l} u_{i} \vec{\nabla} u_{r} \\
& +\sum_{(m, j) \in \mathbb{N} \times \mathscr{E}_{\varphi}} c_{m, j}^{k} \varphi^{m} u_{j} \vec{\nabla} \varphi+\sum_{s \in\{1, \ldots, \mu-1\}} \bar{c}_{s}^{k} \vec{\nabla} u_{s} .
\end{aligned}
$$

(Notice that only a finite number of $c_{l, i}^{k_{0}}$ and $\bar{c}_{r}^{k_{0}}$ are nonzero for every $k_{0} \in \mathbb{N}^{*}$.) We must verify (see Equation (7)) that

$$
\delta_{\varphi}^{2}\left(\pi_{n+1}\right)=\frac{1}{2} \sum_{\substack{i+j=n+1 \\ i, j \geq 1}}\left[\pi_{i}, \pi_{j}\right]_{S} \quad \text { for every } n \in \mathbb{N}
$$

For $n=0$, it becomes $\delta_{\varphi}^{2}\left(\pi_{1}\right)=0$ and, according to (30), we have

$$
\pi_{1}=\sum_{(m, j) \in \mathbb{N} \times \mathscr{E}_{\varphi}} c_{m, j}^{1} \varphi^{m} u_{j} \vec{\nabla} \varphi+\sum_{s \in\{1, \ldots, \mu-1\}} \bar{c}_{s}^{1} \vec{\nabla} u_{s}
$$

which is an element of $Z^{2}\left(\mathscr{A},\{\cdot, \cdot\}_{\varphi}\right)$. Now, assume that $n \geq 1$ and let us prove that the skew-symmetric biderivations $\pi_{1}, \pi_{2}, \ldots, \pi_{n+1}$ defined by (30) satisfy (31). By using (27), one obtains that $\frac{1}{2} \sum_{i+j=n+1, i, j \geq 1}\left[\pi_{i}, \pi_{j}\right]_{S}$ consists of six types of sums:

$$
\begin{aligned}
& \frac{1}{2} \sum c_{l, i}^{a} \bar{c}_{r}^{b} c_{m, j}^{c} \bar{c}_{s}^{d}\left[\varphi^{l} u_{i} \vec{\nabla} u_{r}, \varphi^{m} u_{j} \vec{\nabla} u_{s}\right]_{S}, \\
& \frac{1}{2} \sum c_{l, i}^{a} \bar{c}_{r}^{b} c_{m, j}^{q}\left[\varphi^{l} u_{i} \vec{\nabla} u_{r}, \varphi^{m} u_{j} \vec{\nabla} \varphi\right]_{S}, \\
& \frac{1}{2} \sum c_{l, i}^{c} \bar{c}_{r}^{d} c_{m, j}^{p}\left[\varphi^{l} u_{i} \vec{\nabla} u_{r}, \varphi^{m} u_{j} \vec{\nabla} \varphi\right]_{S}, \\
& \frac{1}{2} \sum \bar{c}_{r}^{q} c_{l, i}^{a} \bar{c}_{s}^{b}\left[\varphi^{l} u_{i} \vec{\nabla} u_{r}, \vec{\nabla} u_{s}\right]_{S}, \\
& \frac{1}{2} \sum c_{l, i}^{c} \bar{c}_{r}^{d} \bar{c}_{s}^{p}\left[\varphi^{l} u_{i} \vec{\nabla} u_{r}, \vec{\nabla} u_{s}\right]_{S}, \\
& \frac{1}{2} \sum\left(c_{l, i}^{p} \bar{c}_{r}^{q}+c_{l, i}^{q} \bar{c}_{r}^{p}\right)\left[\varphi^{l} u_{i} \vec{\nabla} \varphi, \vec{\nabla} u_{r}\right]_{S},
\end{aligned}
$$

where the sums are taken over indices $a, b, c, d, p, q, r, s, l, m, i, j \in \mathbb{N}$ satisfying

$$
\begin{array}{rlrl}
p+q & =n+1, & a+b=p, & c+d=q, \quad a, b, c, d, p, q \geq 1, \\
l, m \in \mathbb{N}, & i, j \in \mathscr{E}_{\varphi}, & 1 \leq r, s \leq \mu-1 .
\end{array}
$$


Observe that, for any such set of indices, the primed indices defined by

$$
\begin{array}{lll}
p^{\prime}=b+c, & a^{\prime}=c, & i^{\prime}=j, \\
q^{\prime}=a+d, & b^{\prime}=b, & j^{\prime}=i, \\
r^{\prime}=r, & c^{\prime}=a, & l^{\prime}=m, \\
s^{\prime}=s, & d^{\prime}=d, & m^{\prime}=l
\end{array}
$$

satisfy the same conditions, so that, in the first sum in (32), one finds the element

$$
c_{l, i}^{a} \bar{c}_{r}^{b} c_{m, j}^{c} \bar{c}_{s}^{d}\left[\varphi^{l} u_{i} \vec{\nabla} u_{r}, \varphi^{m} u_{j} \vec{\nabla} u_{s}\right]_{S}
$$

and the element

$$
c_{l^{\prime}, i^{\prime}}^{a^{\prime}} \bar{c}_{r^{\prime}}^{b^{\prime}} c_{m^{\prime}, j^{\prime}}^{c^{\prime}} \bar{c}_{s^{\prime}}^{d^{\prime}}\left[\varphi^{l^{\prime}} u_{i^{\prime}} \vec{\nabla} u_{r^{\prime}}, \varphi^{m^{\prime}} u_{j^{\prime}} \vec{\nabla} u_{s^{\prime}}\right]_{S} .
$$

By definition of the primed indices, this second term is then equal to the element $c_{l, i}^{a} \bar{c}_{r}^{b} c_{m, j}^{c} \bar{c}_{s}^{d}\left[\varphi^{m} u_{j} \vec{\nabla} u_{r}, \varphi^{l} u_{i} \vec{\nabla} u_{s}\right]_{S}$, whose sum with (33) is zero, according to (26). This fact proves that the first sum in (32) is equal to zero. With analogous arguments, one finds that the next four sums in (32) are also zero. We have then found that $\frac{1}{2} \sum_{i+j=n+1, i, j \geq 1}\left[\pi_{i}, \pi_{j}\right]_{S}$ is given by the last sum in (32), that is,

$$
\frac{1}{2} \sum_{\substack{i+j=n+1 \\ i, j \geq 1}}\left[\pi_{i}, \pi_{j}\right]_{S}=\frac{1}{2} \sum_{\substack{(l, i) \in \mathbb{N} \times \mathscr{E}_{\varphi} \\ r \in\{1, \ldots, \mu-1\}}} \sum_{\substack{p+q=n+1 \\ p, q \in \mathbb{N}^{*}}}\left(c_{l, i}^{p} \bar{c}_{r}^{q}+c_{l, i}^{q} \bar{c}_{r}^{p}\right)\left[\varphi^{l} u_{i} \vec{\nabla} \varphi, \vec{\nabla} u_{r}\right]_{S}
$$

$$
=\sum_{\substack{(l, i) \in \mathbb{N} \times \mathscr{C}_{\varphi} \\ r \in\{1, \ldots, \mu-1\}}} \sum_{\substack{p+q=n+1 \\ p, q \in \mathbb{N}^{*}}} c_{l, i}^{p} \bar{c}_{r}^{q} \delta_{\pi_{0}}^{2}\left(\varphi^{l} u_{i} \vec{\nabla} u_{r}\right),
$$

where, for the second equality, we have used (28). Now, let us consider $\delta_{\pi_{0}}^{2}\left(\pi_{n+1}\right)$. According to Equation (30), for $k=n+1$, and Lemma 3.2,

$$
\pi_{n+1} \in \sum_{\substack{(l, i) \in \mathbb{N} \times \mathscr{E}_{\varphi} \\ r \in\{1, \ldots, \mu-1\}}} \sum_{\substack{p+q=n+1 \\ p, q \in \mathbb{N}^{*}}} c_{l, i}^{p} \bar{c}_{r}^{q} \varphi^{l} u_{i} \vec{\nabla} u_{r}+Z^{2}\left(\mathscr{A},\{\cdot, \cdot\}_{\varphi}\right) .
$$

Combining the equations (34) and (35), we obtain that (31) holds, hence parts (a) and (b). For part (c), we use [Pichereau 2006, Proposition 4.5] to obtain that, if $\varpi(\varphi) \neq|\varpi|$, then $H^{1}\left(\mathscr{A},\{\cdot, \cdot\}_{\varphi}\right)$ is zero, and we conclude with the help of Proposition 2.7. Part (d) follows finally from the fact that Propositions 2.2 and 2.7 are also valid for $m$-th order deformations.

This proposition leads to the following result:

Corollary 3.5. Let $\varphi \in \mathbb{F}[x, y, z]$ be a weight-homogeneous polynomial with an isolated singularity. Then, for all $m \in \mathbb{N}^{*}$, every $m$-th order deformation of $\{\cdot, \cdot\}_{\varphi}$ extends to a $(m+1)$-st order deformation of $\{\cdot, \cdot\}_{\varphi}$. 
Proof. According to Proposition 3.3(d), any $m$-th order deformation $\pi_{(m)}^{\prime}$ of $\{\cdot, \cdot\}_{\varphi}$ is equivalent to an $m$-th order deformation of the form $\pi_{(m)}:=\{\cdot, \cdot\}_{\varphi}+\sum_{n=1}^{m} \pi_{n} \nu^{n}$, where the $\pi_{n}$ are defined as in (29). Let us denote by $\Phi: \mathcal{A}^{v} /\left\langle v^{m+1}\right\rangle \rightarrow \mathscr{A}^{v} /\left\langle v^{m+1}\right\rangle$, the equivalence morphism from $\pi_{(m)}$ to $\pi_{(m)}^{\prime}$. Let us extend $\Phi$ to an automorphism of $\left(\mathscr{A}^{v} /\left\langle v^{m+2}\right\rangle, \cdot\right)$, in a natural way.

According to Proposition 3.3, $\pi_{(m+1)}:=\{\cdot, \cdot\}_{\varphi}+\sum_{n=1}^{m+1} \pi_{n} \nu^{n}$, where $\pi_{m+1}$ is defined with the analogue of (29), extends $\pi_{(m)}$ as an $(m+1)$-st order deformation. Then, the $(m+1)$-st order deformation $\pi_{(m+1)}^{\prime}$ defined by the formula

$$
\pi_{(m+1)}^{\prime}[F, G]=\Phi\left(\pi_{(m+1)}\left[\Phi^{-1}(F), \Phi^{-1}(G)\right]\right) \bmod v^{m+2}
$$

(for $F, G \in \mathscr{A}$ or $F, G \in \mathscr{A}^{v} /\left\langle v^{m+2}\right\rangle$ ) extends $\pi_{(m)}^{\prime}$ also as an $(m+1)$-st order deformation.

In general, this property of extendibility of deformations is not satisfied by an arbitrary Poisson structure; the particular family of Poisson algebras associated to weight-homogeneous polynomials with an isolated singularity $\left(\mathscr{A},\{\cdot, \cdot\}_{\varphi}\right)$ has specific and nice properties of deformations.

Let us now consider the particular case where $\varpi(\varphi)=|\varpi|$, for which we have $H^{1}\left(\mathscr{A},\{\cdot, \cdot\}_{\varphi}\right) \simeq \mathbb{F}[\varphi] \vec{e}_{\varpi}$ according to [Pichereau 2006, Proposition 4.5]. In this case, Proposition 3.3(c) and the uniqueness of the constants $c_{l, i}^{k}$ and $\bar{c}_{r}^{k}$ do not hold anymore. In particular, we will see that $\Phi=\exp \left(\vec{e}_{\varpi} v\right)$, which is an algebra morphism $\mathscr{A}^{v} \rightarrow \mathscr{A}^{v}$ equal to the identity modulo $v$, is always an equivalence morphism between two different (except in a very particular case) formal deformations of the family given in Proposition 3.3. To see that, assume $\varpi(\varphi)=|\varpi|$ and define

$$
\xi:=\vec{e}_{\varpi} v=\varpi_{1} x v \frac{\partial}{\partial x}+\varpi_{2} y v \frac{\partial}{\partial y}+\varpi_{3} z \nu \frac{\partial}{\partial z} \in \mathfrak{X}_{0}^{1}\left(\mathscr{A}^{v}\right) .
$$

Then take the formal deformation $\pi_{*}$ of $\pi_{0}=\{\cdot, \cdot\}_{\varphi}$ given by two arbitrary families of constants

$$
\left(c_{l, i}^{a}\right)_{(l, i) \in \mathbb{N} \times \mathscr{\complement}_{\varphi}, a \in \mathbb{N}^{*}} \quad \text { and } \quad\left(\bar{c}_{r}^{b}\right)_{1 \leq r \leq \mu-1, b \in \mathbb{N}^{*}}
$$

(with only a finite number of nonzero $c_{l, i}^{a_{0}}$ and $\bar{c}_{r}^{b_{0}}$ for every $a_{0}, b_{0} \in \mathbb{N}^{*}$ ) and by Equation (29). Let us denote by $\pi_{*}^{\prime}$ the formal deformation of $\pi_{0}$ given by $\pi_{*}^{\prime}:=e^{\operatorname{ad} d_{\xi}}\left(\pi_{*}\right)$. According to Lemma 2.1 , the deformation $\pi_{*}^{\prime}$ is equivalent to $\pi_{*}$ and $\Phi=e^{\xi}$ is an equivalence morphism from $\pi_{*}$ to $\pi_{*}^{\prime}$. Then a direct computation (using the Euler formula (22)) shows that $\pi_{*}^{\prime}$ is also given by $\pi_{*}^{\prime}=\pi_{0}+\sum_{n \in \mathbb{N}^{*}} \pi_{n}^{\prime} \nu^{n}$, where, for all $n \in \mathbb{N}^{*}$,

$$
\begin{aligned}
\pi_{n}^{\prime}= & \sum_{\substack{(l, i) \in \mathbb{N} \times \mathscr{E}_{\varphi} \\
s \in\{1, \ldots, \mu-1\}}} \sum_{\substack{a+b=n \\
a, b \in \mathbb{N}^{*}}} c_{l, i}^{\prime a} \bar{c}_{s}^{\prime b} \varphi^{l} u_{i} \vec{\nabla} u_{s} \\
& +\sum_{(m, j) \in \mathbb{N} \times \mathscr{E}_{\varphi}} c_{m, j}^{\prime n} \varphi^{m} u_{j} \vec{\nabla} \varphi+\sum_{s \in\{1, \ldots, \mu-1\}} \bar{c}_{s}^{\prime n} \vec{\nabla} u_{s},
\end{aligned}
$$


with, for $n \in \mathbb{N}^{*},(l, i) \in \mathbb{N} \times \mathscr{E}_{\varphi}$ and $1 \leq r \leq \mu-1$,

$$
c_{l, i}^{\prime n}:=\sum_{\substack{k+r=n \\ k, r \in \mathbb{N}^{*}}} \frac{1}{r !} c_{l, i}^{k}\left(|\varpi| l+\varpi\left(u_{i}\right)\right)^{r}, \quad \bar{c}_{s}^{\prime n}:=\sum_{\substack{k+r=n \\ k, r \in \mathbb{N}^{*}}} \frac{1}{r !} \bar{c}_{s}^{k}\left(\varpi\left(u_{s}\right)-|\varpi|\right)^{r} .
$$

Moreover, $\pi_{*}^{\prime}=\pi_{*}$ if and only if $c_{l, i}^{n}=0$ for all $(l, i) \in\left(\mathbb{N} \times \mathscr{E}_{\varphi}\right)-\{(0,0)\}$, and $\bar{c}_{s}^{k}=0$ for all $1 \leq s \leq \mu-1$ such that $\varpi\left(u_{s}\right) \neq|\varpi|$. Thus, $\pi_{*}^{\prime}=\pi_{*}$ if and only if $\pi_{*}$ is of the form

$$
\pi_{*}=\pi_{0}+\sum_{n \in \mathbb{N}^{*}}\left(\sum_{\substack{a+b=n \\ a, b \in \mathbb{N}^{*}}} \sum_{\substack{s=1 \\ \varpi \\\left(u_{s}\right)=|\varpi|}}^{\mu-1} c_{0,0}^{a} \bar{c}_{s}^{b} \vec{\nabla} u_{s}+c_{0,0}^{n} \vec{\nabla} \varphi+\sum_{\substack{t=1 \\ \omega\left(u_{t}\right)=|\varpi|}}^{\mu-1} \bar{c}_{t}^{n} \vec{\nabla} u_{t}\right) v^{n},
$$

that is, $\pi_{*}$ is a weight-homogeneous formal deformation of $\pi_{0}$ of (weighted) degree zero, or, in other words, each $\pi_{n}$ is a weight-homogeneous biderivation of weighted degree zero for all $n \in \mathbb{N}$. (For more information about weight-homogeneous biderivations, see [Laurent-Gengoux et al. $\geq 2008$ ]).

3.4. Properties of the formal deformations of $\{\cdot, \cdot\}_{\varphi}$. Since in Proposition 3.3 we have obtained an explicit expression for the formal deformations of the Poisson bracket $\{\cdot, \cdot\}_{\varphi}$, we will now be able to give some properties of these deformations when $\varphi \in \mathbb{F}[x, y, z]$ is assumed to be weight-homogeneous with an isolated singularity. First, we obtain the following:

Proposition 3.6. Let $\varphi \in \mathscr{A}=\mathbb{F}[x, y, z]$ be a weight-homogeneous polynomial with an isolated singularity. Consider the Poisson algebra $\left(\mathscr{A},\{\cdot, \cdot\}_{\varphi}\right)$ associated to $\varphi$, where $\{\cdot, \cdot\}_{\varphi}$ is the Poisson bracket given by (18). Then, for every formal deformation $\pi_{*}^{\prime}$ of $\{\cdot, \cdot\}_{\varphi}$, there exist $\chi^{v}, \varphi^{v} \in \mathscr{A}^{v}$ such that $\pi_{*}^{\prime}$ is equivalent to the formal deformation $\pi_{*}=\chi^{\nu} \vec{\nabla} \varphi^{\nu}$.

Proof. According to Proposition 3.3, an arbitrary formal deformation $\pi_{*}^{\prime}$ of $\{\cdot, \cdot\}_{\varphi}$ is equivalent to a formal deformation $\pi_{*}$ of the form

$$
\pi_{*}=\{\cdot, \cdot\}_{\varphi}+\sum_{n \in \mathbb{N}^{*}} \pi_{n} v^{n},
$$

with $\pi_{n}$ given by (29) for all $n \in \mathbb{N}^{*}$, where the elements $c_{l, i}^{k}$ and $\bar{c}_{r}^{k}$ (with $k \in \mathbb{N}^{*}$, $(l, i) \in \mathbb{N} \times \mathscr{E}_{\varphi}$ and $1 \leq r \leq \mu-1$ ) are constants in $\mathbb{F}$ (with only a finite number of nonzero $c_{l, i}^{a}$ and $\bar{c}_{r}^{b}$ for every $a, b \in \mathbb{N}^{*}$ ). It is easy to verify that the elements of $\mathscr{A}^{v}$ defined by

$$
\chi^{\nu}:=1+\sum_{a \in \mathbb{N}^{*}}\left(\sum_{(l, i) \in \mathbb{N} \times \mathscr{E}_{\varphi}} c_{l, i}^{a} \varphi^{l} u_{i}\right) v^{a} \quad \text { and } \quad \varphi^{v}:=\varphi+\sum_{b \in \mathbb{N}^{*}}\left(\sum_{r=1}^{\mu-1} \bar{c}_{r}^{b} u_{r}\right) v^{b}
$$


satisfy the identity $\pi_{*}=\chi^{\nu} \vec{\nabla} \varphi^{\nu} \in\left(\mathscr{A}^{\nu}\right)^{3} \simeq \mathfrak{X}^{2}\left(\mathscr{A}^{v}\right)$, so $\pi_{*}^{\prime}$ is equivalent to a deformation of the desired form.

Remark 3.7. It is easy to verify that, on $\mathbb{F}^{3}$, multiplying a Poisson structure $\{\cdot, \cdot\}$ by any polynomial $\chi \in \mathbb{F}[x, y, z]$ gives another Poisson structure $\chi\{\cdot, \cdot\}$. This fact is generally not true in other dimensions. In particular, for every $\chi, \varphi \in \mathbb{F}[x, y, z]$, the skew-symmetric biderivation $\chi\{\cdot, \cdot\}_{\varphi}$ (identified with $\chi \vec{\nabla} \varphi \in \mathscr{A}^{3}$ ) is a Poisson structure on $\mathbb{F}^{3}$. In the Proposition 3.6, we have seen that, morally, if one deforms a Poisson structure of the family $\left(\{\cdot, \cdot\}_{\varphi} \simeq \vec{\nabla} \varphi \mid \varphi \in \mathscr{A}\right)$, one obtains a Poisson structure on $\mathscr{A}^{v}$ that belongs to the family $\left(\chi^{\nu}\{\cdot, \cdot\}_{\varphi^{v}} \simeq \chi^{\nu} \vec{\nabla} \varphi^{\nu} \mid \chi^{\nu}, \varphi^{\nu} \in \mathscr{A}^{\nu}\right)$.

The next corollary gives another property of the formal deformations of $\{\cdot, \cdot\}_{\varphi}$.

Corollary 3.8. Let $\varphi \in \mathscr{A}=\mathbb{F}[x, y, z]$ be a weight-homogeneous polynomial with an isolated singularity. Consider the Poisson algebra $\left(\mathscr{A},\{\cdot, \cdot\}_{\varphi}\right)$ associated to $\varphi$. Every formal deformation of $\{\cdot, \cdot\}_{\varphi}$ admits a formal Casimir.

Proof. First, let us consider a formal deformation of $\pi_{0}$, supposed to be of the form $\pi_{*}=\chi^{\nu} \vec{\nabla} \varphi^{\nu}$, where $\chi^{\nu}, \varphi^{v} \in \mathscr{A}^{\nu}$, and let us show that $\varphi^{\nu}$ is then a formal Casimir for $\pi_{*}$. Under the identifications $\mathfrak{X}^{2}\left(\mathscr{A}^{\nu}\right) \simeq\left(\mathscr{A}^{v}\right)^{3}$ and $\mathfrak{X}^{1}\left(\mathscr{A}^{v}\right) \simeq\left(\mathscr{A}^{v}\right)^{3}$, we indeed have $\pi_{*}\left[\varphi^{\nu}, \cdot\right]=\left(\chi^{\nu} \vec{\nabla} \varphi^{\nu}\right) \times \vec{\nabla} \varphi^{\nu}$, which is equal to zero, since, by writing $\chi^{\nu}=\sum_{i \in \mathbb{N}} \chi_{i} v^{i}$ and $\varphi^{\nu}:=\sum_{j \in \mathbb{N}} \varphi_{j} v^{j}$, where $\chi_{i}, \varphi_{j} \in \mathscr{A}$, we have

$$
\left(\chi^{\nu} \vec{\nabla} \varphi^{\nu}\right) \times \vec{\nabla} \varphi^{\nu}=\sum_{i \in \mathbb{N}} \sum_{l \in \mathbb{N}} \chi_{i}\left(\sum_{j+k=l} \vec{\nabla} \varphi_{j} \times \vec{\nabla} \varphi_{k}\right) v^{i+l},
$$

where, for each $l \in \mathbb{N}$, the sum $\sum_{j+k=l} \vec{\nabla} \varphi_{j} \times \vec{\nabla} \varphi_{k}$ is equal to zero because its summand is skew-symmetric under exchange of $j$ and $k$. Now, according to Proposition 3.6, any formal deformation $\pi_{*}^{\prime}$ of $\{\cdot, \cdot\}_{\varphi}$ is equivalent to a formal deformation of the form $\pi_{*}=\chi^{\nu} \vec{\nabla} \varphi^{\nu}$, where $\chi^{\nu}, \varphi^{\nu} \in \mathscr{A}^{\nu}$. Then there exists a morphism of Poisson algebras $\Phi:\left(\mathscr{A}^{v}, \pi_{*}\right) \rightarrow\left(\mathscr{A}^{v}, \pi_{*}^{\prime}\right)$ that is the identity modulo $\nu$. Thus $\Phi$ is invertible, and we have

$$
\pi_{*}^{\prime}\left[\Phi\left(\varphi^{\nu}\right), F\right]=\Phi\left(\pi_{*}\left[\varphi^{\nu}, \Phi^{-1}(F)\right]\right)=0 \quad \text { for any } F \in \mathscr{A}^{\nu} .
$$

Hence $\Phi\left(\varphi^{\nu}\right)$ is a formal Casimir for $\pi_{*}^{\prime}$.

3.5. The case of singular surfaces in $\mathbb{F}^{3}$. In this last subsection, we study singular surfaces in $\mathbb{F}^{3}$ that are equipped with Poisson structures and are as regular as possible. As in the previous cases, we give an explicit expression for all formal deformations of these Poisson brackets, up to equivalence.

As before, $\varphi \in \mathbb{F}[x, y, z]$ denotes a weight-homogeneous polynomial with an isolated singularity, and the weights of the three variables $x, y, z$ are still denoted by $\varpi_{1}, \varpi_{2}, \varpi_{3}$, and their sum is $|\varpi|=\varpi_{1}+\varpi_{2}+\varpi_{3}$. To such a polynomial, one can associate a surface $\mathscr{F}_{\varphi}$ in $\mathbb{F}^{3}$ whose singular locus is exactly the set on which 
all three partial derivatives of $\varphi$ vanish. In fact, this singular surface is given by the zero locus of $\varphi$, that is, $\mathscr{F}_{\varphi}:\{\varphi=0\}$. This affine space is equipped with its algebra of regular functions $\mathscr{A}_{\varphi}:=\mathbb{F}[x, y, z] /\langle\varphi\rangle$.

In Remark 3.1, we pointed out that $\varphi$ is a Casimir for the Poisson structure $\{\cdot, \cdot\}_{\varphi}$ defined in (18), that is, it is an element of the center of the bracket $\{\cdot, \cdot\}_{\varphi}$. Hence, the Poisson bracket $\{\cdot, \cdot\}_{\varphi}$ goes to the quotient algebra $\mathscr{A}_{\varphi}$, and it induces a bracket $\{\cdot, \cdot\}_{\mathscr{A}_{\varphi}}$ on $\mathscr{A}_{\varphi}$ that is obviously a Poisson bracket.

This subsection studies the formal deformations of this Poisson structure. First, as proved in [Pichereau 2006, Proposition 5.2], we have $\mathfrak{X}^{3}\left(\mathscr{A}_{\varphi}\right) \simeq\{0\}$, so that $H^{3}\left(\mathscr{A}_{\varphi},\{\cdot, \cdot\}_{\mathscr{A}_{\varphi}}\right) \simeq\{0\}$. By the equations (7) that govern the extendibility of deformations, every $m$-th order deformation $\{\cdot, \cdot\}_{\mathscr{A}_{\varphi}}+\pi_{1} v+\cdots+\pi_{m} v^{m}$ of $\{\cdot, \cdot\}_{\mathscr{A}_{\varphi}}$ (with $m \in \mathbb{N}^{*}$ ) extends to deformation $\{\cdot, \cdot\}_{\mathcal{A}_{\varphi}}+\pi_{1} v+\cdots+\pi_{m} v^{m}+\pi_{m+1} v^{m+1}$ of order $m+1$ by choosing for $\pi_{m+1}$ any Poisson 2-cocycle of $\left(\mathscr{A}_{\varphi},\{\cdot, \cdot\}_{\mathscr{A}_{\varphi}}\right)$.

In [Pichereau 2006, Proposition 5.6], we showed that the family

$$
\left\{\wp\left(u_{j} \vec{\nabla} \varphi\right)\left|0 \leq j \leq \mu-1, \varpi\left(u_{j}\right)=\varpi(\varphi)-\right| \varpi \mid\right\},
$$

where $\mu$ is the Milnor number of $\varphi$ and $\wp: \mathbb{E}[x, y, z] \rightarrow \mathscr{A}_{\varphi}$ is the natural projection, gives an $\mathbb{F}$-basis of the second Poisson cohomology space of $\left(\mathscr{A}_{\varphi},\{\cdot, \cdot\}_{\mathscr{A}_{\varphi}}\right)$. Since $H^{3}\left(\mathscr{A}_{\varphi},\{\cdot, \cdot\}_{\mathscr{A}_{\varphi}}\right) \simeq\{0\}$, a simple case of Proposition 2.2, in which the skewsymmetric biderivations $\Psi_{n}^{a}$ can be chosen to be zero, leads to the following result (which is also valid for $m$-th order deformations of $\{\cdot, \cdot\}_{\mathscr{A}_{\varphi}}$ ).

Proposition 3.9. Let $\varphi \in \mathbb{F}[x, y, z]$ be a weight-homogeneous polynomial with an isolated singularity. Consider the Poisson algebra $\left(\mathscr{A}_{\varphi},\{\cdot, \cdot\}_{\mathscr{A}_{\varphi}}\right)$, and write $\mathscr{K}=\left\{j \in\{0, \ldots, \mu-1\}\left|\varpi\left(u_{j}\right)=\varpi(\varphi)-\right| \varpi \mid\right\}$. We have the following:

(a) For every family of constants $\left(\alpha_{j}^{n} \in \mathbb{F}\right)_{j \in \mathscr{K}, n \in \mathbb{N}^{*}}$, the formula

$$
\pi_{*}=\{\cdot, \cdot\}_{\mathscr{A}_{\varphi}}+\sum_{n \in \mathbb{N}^{*}}\left(\sum_{\substack{j=0 \\ \varpi\left(u_{j}\right)=\omega(\varphi)-|\varpi|}}^{\mu-1} \alpha_{j}^{n} \wp\left(u_{j} \vec{\nabla} \varphi\right)\right) v^{n}
$$

defines a formal deformation of $\{\cdot, \cdot\}_{\mathscr{A}_{\varphi}}$.

(b) For any formal deformation $\pi_{*}^{\prime}$ of $\{\cdot, \cdot\}_{\mathscr{A}_{\varphi}}$, there exists a family of constants

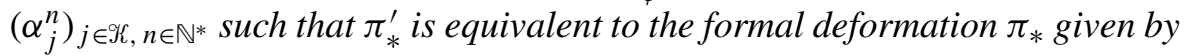
formula (36).

Remark 3.10. According to [Pichereau 2006, Proposition 5.5], we have

$$
H^{1}\left(\mathscr{A}_{\varphi},\{\cdot, \cdot\}_{\mathcal{A}_{\varphi}}\right) \simeq \bigoplus_{\substack{j=0 \\ \varpi\left(u_{j}\right)=\varpi(\varphi)-|\varpi|}}^{\mu-1} \mathbb{F} \wp\left(u_{j} \vec{e}_{\varpi}\right),
$$


which is zero if and only if $H^{2}\left(\mathscr{A}_{\varphi},\{\cdot, \cdot\}_{\mathscr{A}_{\varphi}}\right)$ is also. According to Proposition 3.9, all formal deformations of $\{\cdot, \cdot\}_{\mathbb{A}_{\varphi}}$ are in this case trivial (that is, they are equivalent to $\{\cdot, \cdot\}_{\mathscr{A}_{\varphi}}$ ). In the previous case, considered in Section 3.3, we considered the algebra morphism $\Phi=\exp \left(\vec{e}_{\varpi} v\right)$ in the case that the Euler derivation $\vec{e}_{\varpi}$ defines a nontrivial cohomological class in the first Poisson cohomology space. Here, the derivation $\vec{e}_{\varpi}$ defines such a nontrivial class if and only if $\varpi(\varphi)=|\varpi|$, but, in this case, according to Proposition 3.9, all formal deformations of $\{\cdot, \cdot\}_{\mathscr{A}_{\varphi}}$ are equivalent to a formal deformation of the form

$$
\pi_{*}=\{\cdot, \cdot\}_{\mathcal{A}_{\varphi}}+\sum_{n \in \mathbb{N}^{*}} \alpha_{0}^{n} \wp(\vec{\nabla} \varphi) \nu^{n},
$$

where $\alpha_{0}^{n} \in \mathbb{F}$ for all $n \in \mathbb{N}^{*}$. Also the algebra morphism $\Phi=e^{\xi}$ defined above (with $\xi:=\vec{e}_{\varpi} v$ ) is an equivalence morphism from such a $\pi_{*}$ to

$$
\pi_{*}^{\prime}:=e^{\operatorname{ad}_{\xi}}\left(\pi_{*}\right)=\{\cdot, \cdot\}_{A_{\varphi}}
$$

because $\left[\vec{e}_{\varpi}, \vec{\nabla} \varphi\right]_{S}=0$. Thus, if $\varpi(\varphi)=|\varpi|$, the Poisson structure $\{\cdot, \cdot\}_{\mathscr{A}_{\varphi}}$ is rigid, that is, all its formal deformations are equivalent to $\{\cdot, \cdot\}_{\mathscr{A}_{\varphi}}$ itself.

Remark 3.11. We may study in the same way the limit case in which the surface in $\mathbb{F}^{3}$ is the plane $\mathbb{F}^{2}$ equipped with its algebra of polynomial functions $\mathbb{F}[x, y]$. Every Poisson structure is in this case of the form

$$
\{\cdot, \cdot\}^{\psi}=\psi \frac{\partial}{\partial x} \wedge \frac{\partial}{\partial y} \quad \text { with } \psi \in \mathbb{F}[x, y] .
$$

In [Monnier 2002], one finds explicit bases for the Poisson cohomology spaces in dimension two for the germified case, while, in [Roger and Vanhaecke 2002], one finds the dimensions of the Poisson cohomology spaces of the Poisson variety $\left(\mathbb{F}[x, y],\{\cdot, \cdot\}^{\psi}\right)$, in the algebraic setting.

We now suppose that the polynomial $\psi \in \mathbb{F}[x, y]$ is a weight-homogeneous polynomial of (weighted) degree $\varpi(\psi)$ associated to the weights of the two variables $x$ and $y$, denoted respectively by $\varpi_{1}$ and $\varpi_{2}$. The methods used in [Monnier 2002] can be applied in the algebraic context. In particular they lead, when $\psi \in \mathbb{E}[x, y]$ is a weight-homogeneous square-free polynomial, to the result that

$$
H^{2}\left(\mathbb{F}[x, y],\{\cdot, \cdot\}^{\psi}\right) \simeq \mathbb{F}[x, y]_{N(\psi)}\{\cdot, \cdot\}^{\psi} \oplus \frac{\mathbb{F}[x, y]}{\left\langle\frac{\partial \psi}{\partial x}, \frac{\partial \psi}{\partial y}\right\rangle} \frac{\partial}{\partial x} \wedge \frac{\partial}{\partial y},
$$

where $\mathbb{F}[x, y]_{N(\psi)}$ is the $\mathbb{F}$-vector space of all weight-homogeneous polynomials in $\mathbb{F}[x, y]$ of (weighted) degree equal to $N(\psi):=\varpi(\psi)-\varpi_{1}-\varpi_{2}$. As in the case of the Poisson algebra $\left(\mathscr{A}_{\varphi},\{\cdot, \cdot\}_{\mathscr{A}_{\varphi}}\right)$, this explicit basis leads to an explicit writing of the formal $m$-th order deformations of $\{\cdot, \cdot\}^{\psi}$. 


\section{Final remarks}

First, we recall the result of $\mathrm{M}$. Kontsevich, stated in the introduction and saying that for a Poisson manifold $(M,\{\cdot, \cdot\})$ there is a correspondence between the equivalence classes of the formal deformations of $\{\cdot, \cdot\}$ and those of the associative products of $\mathscr{F}(M)$ that have as a first order term the Poisson bracket $\{\cdot, \cdot\}$. Considering this, a natural extension of the results given here would be to consider the equivalence classes of the formal deformations of the associative algebra $\mathscr{A}=\mathbb{F}[x, y, z]$ that have as first order term a Poisson bracket of the form $\{\cdot, \cdot\}_{\varphi}$, with $\varphi \in \mathscr{A}$, and compare them to the equivalence classes of the formal deformations of the Poisson structure $\{\cdot, \cdot\}_{\varphi}$ obtained in this paper. We hope to come back to this in a future publication.

Second, after obtaining these results of deformation of the Poisson structures of the form $\{\cdot, \cdot\}_{\varphi}$ for $\varphi \in \mathbb{F}[x, y, z]$, B. Fresse pointed out to me that they could come from an $L_{\infty}$-equivalence between two $L_{\infty}$-algebras. This other point of view opens new perspectives of research, which we plan to explore in the future.

Finally, in [2007], P. Etingof and V. Ginzburg consider "deformations" of Poisson algebras, but with the meaning that the associative product and the Poisson bracket are simultaneously deformed. To do that, they use the notion of "Poisson cohomology" defined in [Fresse 1998; 2006; Ginzburg and Kaledin 2004]; this notion is different from the one used here and in [Pichereau 2006]. It would be interesting to compare this paper with P. Etingof and V. Ginzburg's.

\section{Acknowledgments}

I wish to take this opportunity to thank P. Vanhaecke for drawing my attention to these questions about deformations and for useful discussions about this subject. I also would like to thank B. Fresse, C. Laurent-Gengoux, M. Penkava, R. Yu and N. T. Zung for valuable conversations that contributed to this paper. The hospitality of the University of Antwerp and of the CRM (Centre de Recerca Matematicà, Barcelona) is also greatly acknowledged.

\section{References}

[Bayen et al. 1978a] F. Bayen, M. Flato, C. Fronsdal, A. Lichnerowicz, and D. Sternheimer, "Deformation theory and quantization, I: Deformations of symplectic structures", Ann. Physics 111:1 (1978), 61-110. MR 58 \#14737a Zbl 0377.53024

[Bayen et al. 1978b] F. Bayen, M. Flato, C. Fronsdal, A. Lichnerowicz, and D. Sternheimer, "Deformation theory and quantization, II: Physical applications", Ann. Physics 111:1 (1978), 111-151. MR 58 \#14737b Zbl 0377.53025

[Damianou et al. 2007] P. A. Damianou, H. Sabourin, and P. Vanhaecke, “Transverse Poisson structures to adjoint orbits in semisimple Lie algebras”, Pacific Jour. Math. 232:1 (2007), 111-138. MR 2358034 
[Etingof and Ginzburg 2007] P. Etingof and V. Ginzburg, "Noncommutative del Pezzo surfaces and Calabi-Yau algebras", preprint, 2007. arXiv 0709.3593v2

[Fresse 1998] B. Fresse, "Homologie de Quillen pour les algèbres de Poisson", C. R. Acad. Sci. Paris Sér. I Math. 326:9 (1998), 1053-1058. MR 99j:16001 Zbl 0922.17014

[Fresse 2006] B. Fresse, "Théorie des opérades de Koszul et homologie des algèbres de Poisson", Ann. Math. Blaise Pascal 13:2 (2006), 237-312. MR 2275449 Zbl 1141.55006

[Ginzburg and Kaledin 2004] V. Ginzburg and D. Kaledin, "Poisson deformations of symplectic quotient singularities", Adv. Math. 186:1 (2004), 1-57. MR 2005h:32072 Zbl 1062.53074

[Gutt 2000] S. Gutt, "Variations on deformation quantization", pp. 217-254 in Conférence Moshé Flato 1999, I (Dijon), edited by G. Dito and D. Sternheimer, Math. Phys. Stud. 21, Kluwer Acad. Publ., Dordrecht, 2000. MR 2002a:53119 Zbl 0997.53068

[Huebschmann 1990] J. Huebschmann, "Poisson cohomology and quantization", J. Reine Angew. Math. 408 (1990), 57-113. MR 92e:17027 Zbl 0699.53037

[Laurent-Gengoux et al. $\geq 2008$ ] C. Laurent-Gengoux, A. Pichereau, and P. Vanhaecke, An invitation to Poisson structures, monograph in preparation.

[Lichnerowicz 1977] A. Lichnerowicz, "Les variétés de Poisson et leurs algèbres de Lie associées", J. Differential Geometry 12:2 (1977), 253-300. MR 58 \#18565 Zbl 0405.53024

[Monnier 2002] P. Monnier, "Poisson cohomology in dimension two", Israel J. Math. 129 (2002), 189-207. MR 2003h:53117 Zbl 1077.17018

[Pichereau 2006] A. Pichereau, "Poisson (co)homology and isolated singularities", J. Algebra 299:2 (2006), 747-777. MR 2007k:17026 Zbl 1113.17009

[Roger and Vanhaecke 2002] C. Roger and P. Vanhaecke, "Poisson cohomology of the affine plane", J. Algebra 251:1 (2002), 448-460. MR 2003g:17031 Zbl 0998.17023

[Sternheimer 1998] D. Sternheimer, "Deformation quantization: Twenty years after", pp. 107-145 in Particles, fields, and gravitation (Łódź, 1998), edited by J. Rembieliński, AIP Conf. Proc. 453, Amer. Inst. Phys., Woodbury, NY, 1998. MR 2002b:53144 Zbl 0977.53082

Received May 30, 2008.

ANNE PICHEREAU

MAX-PlanCK-InSTITUT FÜR MATHEMATIK

VIVATSGASSE 7

53111 BONN

GERMANY

pichereau@mpim-bonn.mpg.de 